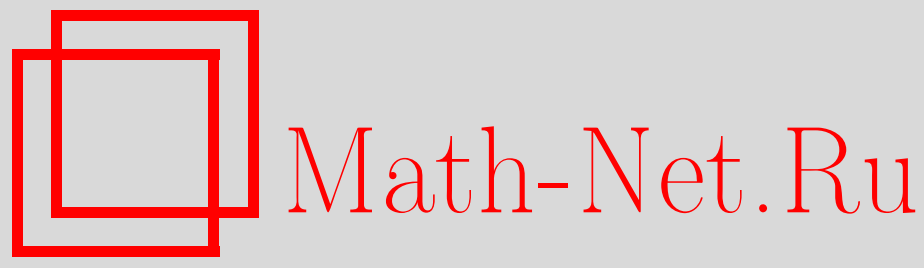

М. С. Ермаков, Асимптотически эффективные статистические выводы для вероятностей умеренных уклонений, Теория вероятн. и ее примен., 2003, том 48, выпуск 4, 676-700

DOI: https://doi.org/10.4213/tvp251

Использование Общероссийского математического портала MathNet.Ru подразумевает, что вы прочитали и согласны с пользовательским соглашением

http://www . mathnet.ru/rus/agreement

Параметры загрузки:

IP : 35.174 .16 .151

26 апреля 2023 г., 17:14:23

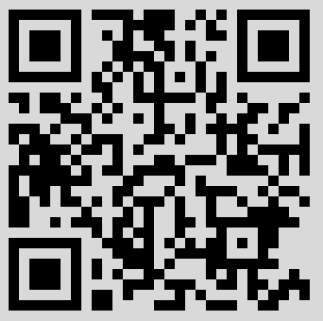


EPMAKOB M. C.*

\section{АСИМПТОТИЧЕСКИ ЭФФЕКТИВНЫЕ СТАТИСТИЧЕСКИЕ ВЫВОДЫ ДЛЯ ВЕРОЯТНОСТЕЙ УМЕРЕННЫХ УКЛОНЕНИЙ}

В работе изучаются нижние границы эффективности для вероятностей умеренных уклонений статистических оценок и критериев. Их доказательство приведено как для логарифмической, так и для точной асимптотики. Для задач проверки гипотез связь нижних границ вероятностей ошибок первого и второго рода выражена через обратную функцию к функции распределения стандартного нормального закона. Нижние границы для вероятностей умеренных уклонений статистических оценок сравнительно легко выводятся из соответствующих границ проверки гипотез.

Ключевые слова и фразы: большие уклонения, умеренные уклонения, эффективность, эффективность по Бахадуру, эффективность по Чернову.

\section{1. Введение}

Пусть $X_{1}, X_{2}, \ldots$ - независимые случайные величины, имеющие одну и ту же вероятностную меру $P_{\theta}$, заданную на сигма-алгебре $B$ множества $S$. Значение параметра $\theta \in \mathbf{R}^{1}$ неизвестно. Для статистических выводов о значении параметра $\theta$ используют обычно некоторую последовательность статистик $T_{n}=T_{n}\left(X_{1}, \ldots, X_{n}\right)$. Асимптотическая эффективность статистик $T_{n}$ традиционно изучается на основе их уклонений от истинного значения параметра $\theta$, имеющих порядок $n^{-1 / 2}$, или на основе вероятностей больших уклонений. Эффективность для уклонений порядка $n^{-1 / 2}\left(\left|T_{n}-\theta\right|>u n^{-1 / 2}>0\right)$ базируется на нижней границе Гаека-Ле Кама в статистическом оценивании и эффективности по Питмену в теории проверки гипотез. Для вероятностей больших уклонений $\left(\left|T_{n}-\theta\right|>u>0\right)$ асимптотическое поведение статистических оценок и тестовых статистик рассматривается относительно эффективностей по Бахадуру [3], Чернову [6] и Ходжесу-Леману [13].

Цель настоящей работы - изучить нижние границы эффективности для промежуточной ситуации умеренных уклонений $\left(T_{n}-\theta>u_{n}\right.$,

* Институт проблем машиноведения РАН, Большой пр. В. О., 61, 199178 С.-Петербург, Россия; e-mail: ermakov@random.ipme.ru 
$u_{n} \rightarrow 0, n u_{n}^{2} \rightarrow \infty$ при $\left.n \rightarrow \infty\right)$. Такие нижние границы будут рассмотрены как для логарифмической, так и для точной асимптотики вероятностей умеренных уклонений. Естественно, нижние границы для точной асимптотики получены для более узкой зоны умеренных уклонений $\left(T_{n}-\theta>u_{n}, n u_{n}^{2} \omega\left(u_{n}\right) \rightarrow 0, n u_{n}^{2} \rightarrow \infty, \omega\left(u_{n}\right) \rightarrow 0\right.$ при $n \rightarrow \infty$, где скорость стремления к нулю функции $\omega$ зависит от свойств регулярности семейства вероятностных мер $P_{\theta}$ ). Анализ оценок в доказательстве нижних границ для точной асимптотики позволяет говорить о том, что условия регулярности, при которых они доказаны, близки к неулучшаемым.

Вероятности умеренных уклонений статистик изучались в целом ряде публикаций (см. [1], [15], [14], [11] и ссылки в этих работах). Однако до последнего времени их эффективность с этих позиций систематически не рассматривалась. Была предложена только промежуточная эффективность по Калленбергу [15], являющаяся аналогом эффективности по Бахадуру для умеренных уклонений тестовых статистик. В последнее десятилетие ситуация существенно изменилась и появился целый ряд работ А. А. Боровкова и А. А. Могульского [4], [5], М. С. Ермакова [7]-[10] и М. Радавичуса [21]. В этих работах были получены нижние границы как для логарифмической, так и для точной асимптотики вероятностей больших уклонений статистических оценок и критериев. Более того, в работах [4], [5] исследован вопрос о нижней границе для следующего члена в асимптотическом разложении вероятностей больших уклонений ошибок первого и второго рода статистических критериев. Нижние границы для точной асимптотики вероятностей больших уклонений статистических оценок и критериев получены при очень жестких условиях регулярности (см. [4], [5], [10]) и цель настоящей работы - получить их при условиях, близких к неулучшаемым. Помимо этого приводятся подробные доказательства и для нижних границ при логарифмической асимптотике, поскольку в предыдущих работах они отсутствовали.

Доказываемые нижние границы в случае задач проверки гипотез основаны на следующем свойстве нормального распределения. Пусть $X_{1}, \ldots, X_{n}$ - выборка независимых одинаково распределенных случайных величин, имеющих нормальное распределение $\mathscr{N}\left(\theta, \sigma^{2}\right)$. Рассмотрим задачу проверки гипотезы $\theta=0$ против альтернативы $\theta=u_{n}$, где $u_{n} \rightarrow 0$ при $n \rightarrow \infty$. Для любого критерия $K_{n}=K_{n u_{n}}=K_{n}\left(X_{1}, \ldots, X_{n}\right)$ проверки гипотезы условимся обозначать его вероятности ошибок первого и второго рода через $\alpha\left(K_{n}\right)=E_{0} K_{n}$ и $\beta\left(K_{n}\right)=\beta\left(K_{n}, u_{n}\right)=$ $E_{u_{n}}\left(1-K_{n}\right)$ соответственно. Обозначим $\bar{X}_{n}=n^{-1}\left(X_{1}+\cdots+X_{n}\right)$ выборочное среднее и определим для него критерии $L_{n}=\chi\left(\bar{X}_{n}>c_{n}\right)$, $0<c_{n}<u_{n}$. Здесь и в дальнейшем мы используем $\chi(D)$ для обозначения индикатора события $D$. Определим функцию стандартного нормального распределения $\Phi(s)=(2 \pi)^{-1 / 2} \int_{-\infty}^{s} \exp \left\{-x^{2} / 2\right\} d x, s \in \mathbf{R}^{1}$. 
Зададим обратную функцию $s=\Phi^{-1}(y)$ уравнением $y=\Phi(s)$. Так как

$$
\Phi^{-1}\left(\alpha\left(L_{n}\right)\right)=-n^{1 / 2} \sigma^{-1} c_{n}, \quad \Phi^{-1}\left(\beta\left(L_{n}\right)\right)=-n^{1 / 2} \sigma^{-1}\left(u_{n}-c_{n}\right),
$$

TO

$$
\Phi^{-1}\left(\alpha\left(L_{n}\right)\right)+\Phi^{-1}\left(\beta\left(L_{n}\right)\right)=-n^{1 / 2} u_{n} \sigma^{-1}
$$

и (1.1) не зависит от выбора значения $c_{n}$, или, другими словами, от выбора $\alpha\left(L_{n}\right)$ или $\beta\left(L_{n}\right)$. Следовательно, для любой последовательности критериев $K_{n}$

$$
\left(n u_{n}^{2} \sigma^{-2}\right)^{-1 / 2}\left(\Phi^{-1}\left(\alpha\left(K_{n}\right)\right)+\Phi^{-1}\left(\beta\left(K_{n}\right)\right)\right) \geqslant-1
$$

и можно высказать предположение, что аналогичная асимптотическая формула

$$
\liminf _{n \rightarrow \infty}\left(n u_{n}^{2} I\right)^{-1 / 2}\left(\Phi^{-1}\left(\alpha\left(K_{n}\right)\right)+\Phi^{-1}\left(\beta\left(K_{n}\right)\right)\right) \geqslant-1
$$

справедлива также и для широкого класса локально асимптотически нормальных семейств распределений $P_{\theta}, \theta \in \mathbf{R}^{1}$, имеющих конечное информационное количество Фишера $I$. Пусть $\alpha\left(K_{n}\right)<c<1$, $\beta\left(K_{n}\right)<c<1$, и пусть $n u_{n}^{2} \rightarrow \infty$ при $n \rightarrow \infty$. Тогда (1.3) может быть представлено в другой форме (см. [4], [5], [7]-[9])

$$
\limsup _{n \rightarrow \infty}\left(n u_{n}^{2} I\right)^{-1 / 2}\left(\left|2 \ln \alpha\left(K_{n}\right)\right|^{1 / 2}+\left|2 \ln \beta\left(K_{n}\right)\right|^{1 / 2}\right) \leqslant 1
$$

Если в (1.4) достигается равенство, то для любой последовательности критериев $N_{n}, \alpha\left(N_{n}\right) \leqslant \alpha\left(K_{n}\right)$, выполнено

$$
\limsup _{n \rightarrow \infty} \ln \beta\left(N_{n}\right)\left[\ln \beta\left(K_{n}\right)\right]^{-1} \leqslant 1 .
$$

Более точный аналог (1.3) имеет следующий вид:

$$
\liminf _{n \rightarrow \infty}\left(n u_{n}^{2} I\right)^{1 / 2}\left\{n^{1 / 2} u_{n} I^{1 / 2}+\Phi^{-1}\left(\alpha\left(K_{n}\right)\right)+\Phi^{-1}\left(\beta\left(K_{n}\right)\right)\right\} \geqslant 0 .
$$

Неравенство (1.6) учитывает следующий член асимптотик $\Phi^{-1}\left(\alpha\left(K_{n}\right)\right)$, $\Phi^{-1}\left(\beta\left(K_{n}\right)\right)$ и, таким образом, представляет собой нижнюю границу для точной асимптотики вероятностей ошибок первого и второго рода. Оно означает, что если в (1.6) достигается равенство, то для любой последовательности критериев $N_{n}, \alpha\left(N_{n}\right) \leqslant \alpha\left(K_{n}\right)$,

$$
\limsup _{n \rightarrow \infty} \beta\left(K_{n}\right)\left[\beta\left(N_{n}\right)\right]^{-1} \leqslant 1 \text {. }
$$


А. А. Боровков и А.А. Могульский [4], [5] назвали нижние границы типа (1.3), (1.4) и (1.6) законами сохранения.

Очевидным следствием нижних границ $(1.3),(1.4)$ и (1.6) является хорошо известный факт. Если для последовательности критериев одна из вероятностей ошибок стремится к нулю, а другая отделена от единицы, т.е. $\alpha\left(K_{n}\right) \rightarrow 0$ при $n \rightarrow \infty$ и $\beta\left(K_{n}\right)<c<1$ или $\alpha\left(K_{n}\right)<c<1$ и $\beta\left(K_{n}\right) \rightarrow 0$ при $n \rightarrow \infty$, то для анализа эффективности статистических критериев нам надо прибегать к постановке задачи о больших или умеренных уклонениях. Если мы рассматриваем задачи проверки гипотез с малыми значениями вероятностей ошибок первого или второго рода $\left(\alpha\left(K_{n}\right) \approx 0.01\right.$ или $\left.\beta\left(K_{n}\right) \approx 0.01\right)$, то применение асимптотических разложений в центральной предельной теореме и неравенства БерриЭссеена показывает, что нормальная аппроксимация корректна при объемах выборки порядка $\approx 5000-10000$ наблюдений. В то же время применение теорем об умеренных уклонениях дает корректность нормальной аппроксимации при числе наблюдений порядка 50 (см. [4]). Та же самая ситуация имеет место и для доверительного оценивания.

Цель настоящей работы - показать, что нижние границы (1.3) и (1.6) справедливы для семейств распределений, удовлетворяющих довольно слабым условиям регулярности, а также на основе неравенств (1.3) и (1.6) сравнительно легко вывести нижние границы для вероятностей умеренных уклонений статистических оценок. Нижняя граница для точной асимптотики вероятностей умеренных уклонений статистических оценок представляет особый интерес. Она может рассматриваться как естественная нижняя граница в доверительном оценивании, когда уровень доверия стремится к единице с ростом объема выборки $n$. Доказано, что для любой последовательности оценок $\hat{\theta}_{n}$, удовлетворяющих определенным условиям регулярности, имеет место

$$
\liminf _{n \rightarrow \infty} \frac{P_{\theta}\left\{\left|\hat{\theta}_{n}-\theta\right|>u_{n}\right\}}{2\left(1-\Phi\left(n^{1 / 2} I^{1 / 2} u_{n}\right)\right)} \geqslant 1
$$

в соответствующей зоне умеренных уклонений $\left(n u_{n}^{2} \rightarrow \infty, n u_{n}^{2} \omega\left(u_{n}\right) \rightarrow 0\right.$ при $n \rightarrow \infty)$. Данное утверждение представляет собой естественное расширение нижней границы для эффективности по Вольфовицу [24] на зону умеренных уклонений. Укажем, что неравенство (1.8) было доказано М. Радавичусом [21] с правой частью, равной $1 /(2 e)$, и автором [10] при существенно более сильных предположениях для многомерного параметра.

На основе предложенных нижних границ нами вводятся два понятия асимптотической эффективности. Последовательность критериев или оценок называется (MD) асимптотически эффективной в смысле вероятностей умеренных уклонений, если на ней достигается нижняя граница для логарифмической асимптотики вероятностей умеренных уклонений. 
Если достигается нижняя граница для точной асимптотики вероятностей умеренных уклонений, то мы будем говорить, что соответствующая последовательность критериев или оценок (SMD) асимптотически эффективна в сильном смысле в соответствующей зоне умеренных уклонений.

MD и SMD эффективности можно рассматривать как естественные расширения традиционных мер эффективности на зону умеренных уклонений (эффективности Питмена в проверке гипотез и локально асимптотически минимаксной эффективности в оценивании). Они показывают, что информация Фишера остается нижней границей и в зоне умеренных уклонений. Как упоминалось выше, существует целый ряд работ, посвященных нормальной аппроксимации для вероятностей умеренных уклонений статистик. Большая часть этих результатов допускает интерпретацию с точки зрения эффективностей умеренных уклонений.

Сушествует прямая связь между MD и другими типами эффективности больших уклонений в задачах проверки гипотез. Промежуточная эффективность по Калленбергу, локальные эффективности по Бахадуру, Чернову и Ходжесу-Леману оказываются частными случаями MD эффективности.

Нижняя граница для промежуточной эффективности по Калленбергу [16] (для нее предполагае'гся дополнительно, что $0<c_{1}<\beta\left(K_{n}\right)<$ $c_{2}<1$ ) непосредственно следует из (1.4):

$$
\limsup _{n \rightarrow \infty}\left(n u_{n}^{2} I\right)^{-1}\left|2 \ln \alpha\left(K_{n}\right)\right| \leqslant 1
$$

Для $u \in(0, \delta), \delta>0$, обозначим $K_{n u}$ последовательности критериев проверки гипотезы $\theta=0$ против альтернативы $\theta=u$. Тогда, так как (1.4) выполнено для любой последовательности $u_{n}, u_{n} \rightarrow 0, n u_{n}^{2} \rightarrow \infty$ при $n \rightarrow \infty$, то имеет место и следующее неравенство:

$$
\limsup _{u \rightarrow 0} \limsup _{n \rightarrow \infty}\left(n u^{2} I\right)^{-1 / 2}\left(\left|2 \ln \alpha\left(K_{n u}\right)\right|^{1 / 2}+\left|2 \ln \beta\left(K_{n u}\right)\right|^{1 / 2}\right) \leqslant 1,
$$

если $\alpha\left(K_{n u}\right)<c<1$ и $\beta\left(K_{n u}\right)<1$.

Отсюда получаем нижнюю границу для локальной эффективности по Бахадуру [3] (предполагается, что $0<c_{1}<\beta\left(K_{n u}\right)<c_{2}<1$ )

$$
\limsup _{u \rightarrow 0} \limsup _{n \rightarrow \infty}\left(n u^{2} I\right)^{-1}\left|2 \ln \alpha\left(K_{n u}\right)\right| \leqslant 1
$$

локальной эффективности по Ходжесу-Леману [13] $\left(0<c_{1}<\alpha\left(K_{n u}\right)<\right.$ $\left.c_{2}<1\right)$

$$
\limsup _{u \rightarrow 0} \limsup _{n \rightarrow \infty}\left(n u^{2} I\right)^{-1}\left|2 \ln \beta\left(K_{n u}\right)\right| \leqslant 1
$$


и локальной эффективности по Чернову [6]

$$
\limsup _{u \rightarrow 0} \limsup _{n \rightarrow \infty}\left(n u_{n}^{2} I\right)^{-1} \min \left\{\left|2 \ln \alpha\left(K_{n u}\right)\right|,\left|2 \ln \beta\left(K_{n u}\right)\right|\right\} \leqslant 1 .
$$

Для $u_{n}=u n^{-1 / 2}$ нижние границы (1.3), (1.6) переходят в нижние границы для эффективности по Питмену. Такая связь эффективностей по Питмену, Бахадуру, Чернову и Ходжесу-Леману была исследована в работах [23] и [17], [18] при более сильных предположениях.

В оценках мы будем использовать большое число различных положительных констант. Условимся их всех обозначать одними и теми же буквами $c, C$.

\section{2. Нижние границы для MD и SMD асимптотических эффективностей}

\section{1. Вероятности умеренных уклонений для критерия от-} ношения правдоподобия. Доказательство всех нижних границ основано только на асимптотической нормальности вероятностей умеренных уклонений логарифма отношения правдоподобия (локальной асимптотической нормальности отношения правдоподобия не требуется). Анализ предельного поведения отношения правдоподобия традиционно осуществляется с использованием техники расстояния Хеллингера (см. [19], $[20],[22])$. Поэтому все результаты в наиболее общей форме будут получены в терминах интеграла Хеллингера. Результаты в терминах информации Фишера легко выводятся из этих общих предположений.

Обозначим $\Lambda$ множество всех вероятностных мер, заданных на сигма-алгебре $B$ множества $S$. Предположим, что вероятностные меры $P_{\theta}, \theta \in \mathbf{R}^{1}$, абсолютно непрерывны относительно меры $\nu \in \Lambda$ и имеют плотности $f(x, \theta)=d P_{\theta} / d \nu(x), x \in S$. Для любых $\theta, t \in \mathbf{R}^{1}$ обозначим $P_{\theta t}^{\mathrm{a}}$ и $P_{\theta t}^{\mathrm{s}}$ соответственно абсолютно непрерывную и сингулярную компоненты меры $P_{\theta}$ относительно меры $P_{t}$. Положим $g(x, u)=$ $g(x, t, u)=(f(x, t+u) / f(x, t))^{1 / 2}-1$ для всех $x \in S, u \in \mathbf{R}^{1}$.

Статистический эксперимент $E=\left\{(S, B), P_{\theta}, \theta \in \mathbf{R}^{1}\right\}$ имеет конечную информацию Фишера при значении параметра $t$, если существует функция $\varphi(x) \in L_{2}\left(P_{t}\right)$ такая, что

$$
\int_{S}(g(x, t, u)-u \varphi(x))^{2} d P_{t}=o\left(u^{2}\right), \quad P_{t+u, t}^{\mathrm{s}}(S)=o\left(u^{2}\right)
$$

при $u \rightarrow 0$. Фишеровская информация равна $I=I(t)=4 \int_{S} \varphi^{2}(x) d P_{t}$.

Для любых вероятностных мер $P_{\theta_{1}}, P_{\theta_{2}}, \theta_{1}, \theta_{2} \in \mathbf{R}^{1}$, определим расстояние Хеллингера

$$
\rho\left(P_{\theta_{1}}, P_{\theta_{2}}\right)=\rho\left(\theta_{1}, \theta_{2}\right)=\left(\int_{S}\left(f^{1 / 2}\left(x, \theta_{1}\right)-f^{1 / 2}\left(x, \theta_{2}\right)\right)^{2} d \nu\right)^{1 / 2}
$$


Нижние границы даны в терминах информации Фишера и расстояния Хеллингера, если выполнены условия А и В соответственно.

А. Существует функция $\omega(u)=\omega(|u|), \omega(u) \rightarrow 0$ при $u \rightarrow 0, \omega(u)>$ $C u, \omega(2 u)<C \omega(u), \omega(u)$ возрастает при $u>0$, такая, что для всех $u$ из некоторой окрестности $t$

$$
\begin{gathered}
\int_{S}(g(x, u)-u \varphi(x))^{2} d P_{t}<C u^{2} \omega(u), \\
\left|4 \rho^{2}(t, t+u)-u^{2} I\right|<C u^{2} \omega(u) \\
\int_{S} \varphi^{2}(x) \omega^{-1}\left(\varphi^{-1}(x)\right) d P_{t}<\infty
\end{gathered}
$$

и $P_{t+u, t}^{\mathrm{s}}(S)=O\left(u^{2} \omega(u)\right)$ при $u \rightarrow 0$.

В частности, если $\omega(u)=|u|^{\gamma}$ и $0<\gamma \leqslant 1$, то (2.4) означает $\int_{S}|\varphi(x)|^{2+\gamma} d P_{t}<\infty$.

В. Существует функция $\omega(u)=\omega(|u|), \omega(u)>C|u|, \omega(2 u)<C \omega(u)$, $\omega(u) \rightarrow 0$ при $u \rightarrow 0, \omega(u)$ возрастает при $u>0$, такая, что для любого $\varepsilon>0$ и всех $u$ из некоторой окрестности $t$

$$
\begin{aligned}
& \int_{S} g^{2}(x, u) \chi(|g(x, u)|>\varepsilon) d P_{t}<C_{\varepsilon} u^{2} \omega(u), \\
& \int_{S} g^{2}(x, u) d P_{t}>C u^{2} \\
& \int_{S} g^{3}(x, u) \chi(|g(x, u)|<\varepsilon) d P_{t}<C_{\varepsilon} u^{2} \omega(u)
\end{aligned}
$$

и $P_{t+u, t}^{\mathbf{s}}(S)=O\left(u^{2} \omega(u)\right)$ при $u \rightarrow 0$.

Отметим, что существование конечной информации Фишера влечет существование функции $\omega$ такой, что имеют место условия $\mathrm{A}$ и В.

Пусть $\omega(u)=|u|^{\gamma}, \gamma>0$, и пусть $g_{\gamma}(x, u)=(f(x, t+u) \times$ $\left.f(x, t)^{-1}\right)^{1 /(2+\gamma)}-1$. Тогда В имеет место, если

$$
C_{1}|u|^{2+\gamma}<\int_{S} g_{\gamma}^{2+\gamma}(x, u) d P_{t}<C_{2}|u|^{2+\gamma}, \quad u \in \mathbf{R}^{1}
$$

и $P_{t+u, t}^{\mathrm{s}}(S)=O\left(|u|^{2+\gamma}\right)$ при $u \rightarrow 0$.

П р и м е р 2.1. Пусть $t=0$ и $f(x-u)=C_{\lambda}|x-u|^{\lambda} \exp \{-|x-u|\}$, где $x \in \mathbf{R}^{1}$ и $\lambda>1$. Тогда условия А и $\mathrm{B}$ имеют место для $\omega(u)=|u|^{\zeta-1}$, $\zeta=\min \{2, \lambda\}$.

П р и м е р 2.2. Пусть $X_{1}, \ldots, X_{n}$ - независимые одинаково распределенные случайные величины, имеющие нормальное распределение. Пусть $E_{\theta} X_{1}=\theta$ для $\theta \geqslant 0$ и $E_{\theta} X_{1}=2 \theta$ для $\theta<0$. Тогда выполнено условие В для $t=0$ при $\gamma=1$. 
Так как из выполнения условия А следует выполнение условия В, во всех результатах, приведенных ниже в терминах расстояния Хеллингера, $\rho_{n}=\rho\left(t, t+u_{n}\right)$ можно положить равным $\left(\frac{1}{4} u_{n}^{2} I\right)^{1 / 2}$, если А имеет место. Это будет постоянно подчеркиваться в утверждениях теорем.

Нами будет показано, что в задачах проверки гипотез нижняя граница точной асимптотики вероятностей умеренных уклонений достигается в более широкой области, если выполнено следующее дополнительное условие.

С. Сушествует $\delta>0$ такое, что для любого $s,-\delta<s<1+\delta$, имеет место

$$
\int_{S} f^{s}(x, t) f^{1-s}(x, t+u) \chi(|g(x, u)|>\varepsilon) d \nu<C_{\varepsilon} u^{2} \omega(u)
$$

для всех $\varepsilon>0$ и любого $u$ из некоторой окрестности $t$.

Легко видеть, что при $\delta=0$ условие $\mathrm{C}$ всегда выполнено, если имеет место (2.5) (см. (3.14)).

Обозначим $\xi_{n i}=\xi_{n i}\left(X_{i}\right)=\ln \left[f\left(X_{i}, t+u_{n}\right) / f\left(X_{i}, t\right)\right], \quad \eta_{n i}=$ $\eta_{n i}\left(t, u_{n}, X_{i}\right)=g\left(X_{i}, t, u_{n}\right), \tau_{i}=\varphi\left(X_{i}\right)$ для $1 \leqslant i \leqslant n$. Определим события $B_{1 n i}=B_{1 n i}(\varepsilon)=\left\{x_{i}:\left|\xi_{n i}\left(x_{i}\right)\right|<\varepsilon, x_{i} \in S\right\}, B_{2 n i}=B_{2 n i}(\varepsilon)=$ $\left\{x_{i}:\left|\eta_{n i}\left(t, u_{n}, x_{i}\right)\right|<\varepsilon, x_{i} \in S\right\}$ и $B_{3 n i}=B_{3 n i}(\varepsilon)=\left\{x_{i}:\left|\varphi\left(x_{i}\right)\right|<\varepsilon u_{n}^{-1}\right.$, $\left.x_{i} \in S\right\}$ для $1 \leqslant i \leqslant n$. В дальнейшем для $i=1$ условимся опускать в обозначениях индекс $i$, т.е. $\xi_{n}=\xi_{n 1}, \eta_{n}=\eta_{n 1}, \tau=\tau_{1}, B_{1 n}=B_{1 n 1}$ и т.д.

Обозначим

$$
\begin{aligned}
N_{n 1} & =\frac{1}{2 n^{1 / 2} \rho_{n}} \sum_{i=1}^{n} \xi_{n i}, \quad N_{n 2}=\frac{1}{n^{1 / 2} \rho_{n}} \sum_{i=1}^{n} \eta_{n i}, \quad N_{n 3}=2 n^{-1 / 2} I^{-1 / 2} \sum_{i=1}^{n} \tau_{i}, \\
N_{n 1 \varepsilon} & =\frac{1}{2 n^{1 / 2} \rho_{n}} \sum_{i=1}^{n} \xi_{n i} \chi\left(B_{1 n i}\right), \quad N_{n 2 \varepsilon}=\frac{1}{n^{1 / 2} \rho_{n}} \sum_{i=1}^{n} \eta_{n i} \chi\left(B_{2 n i}\right), \\
N_{n 3 \varepsilon} & =2 n^{-1 / 2} I^{-1 / 2} \sum_{i=1}^{n} \tau_{i} \chi\left(B_{3 n i}\right) .
\end{aligned}
$$

По сушеству суммы $N_{n s}, N_{n s \varepsilon}, 1 \leqslant s \leqslant 3$, представляют собой различные аппроксимации случайной компоненты логарифма отношения правдоподобия. Их асимптотическое поведение вероятностей больших уклонений приведено в теореме 2.1 .

Для $s=1,2,3$ определим события $U_{n s}=\bigcap_{i=1}^{n} B_{s n i}$.

Теорема 2.1. Пусть выполнены условия В и С. Пусть $u_{n} \rightarrow 0$, $n u_{n}^{2} \rightarrow \infty$ nрu $n \rightarrow \infty$. Пусть $-\left(1+2 \delta_{1}\right) n^{1 / 2} \rho_{n}<C_{n}<\left(1+2 \delta_{1}\right) n^{1 / 2} \rho_{n}$, где $\delta_{1}<\delta$. Тогда

$$
\begin{aligned}
P_{t}\left\{N_{n 1}>C_{n}\right\} & =\Phi\left(-C_{n}+E_{t}\left[N_{n 1}\right]+O\left(n^{1 / 2} u_{n} \omega\left(u_{n}\right)\right)\right) \\
& =\Phi\left(-C_{n}-n^{1 / 2} \rho_{n}+O\left(n^{1 / 2} u_{n} \omega\left(u_{n}\right)\right)\right),
\end{aligned}
$$




$$
\begin{aligned}
P_{t+u_{n}}\left\{N_{n 1}<C_{n}\right\} & =\Phi\left(C_{n}-E_{t+u_{n}}\left[N_{n 1}\right]+O\left(n^{1 / 2} u_{n} \omega\left(u_{n}\right)\right)\right) \\
& =\Phi\left(C_{n}-n^{1 / 2} \rho_{n}+O\left(n^{1 / 2} u_{n} \omega\left(u_{n}\right)\right)\right) .
\end{aligned}
$$

Предположим, что выполнено условие $\mathrm{A}$, если $s=3, u \mathrm{~B}$, если $s=1,2$. Тогда для любой последовательности $C_{n},\left|C_{n}\right|<C n^{1 / 2} \rho_{n}$,

$$
\begin{aligned}
P_{t}\left\{N_{n s}>C_{n}, U_{n s}\right\} & =P_{t}\left\{N_{n s \varepsilon}>C_{n}\right\} \exp \left\{-O\left(n u_{n}^{2} \omega\left(u_{n}\right)\right)\right\} \\
& =\Phi\left(-C_{n}+E_{t}\left[N_{n s \varepsilon}\right]+O\left(n^{1 / 2} u_{n} \omega\left(u_{n}\right)\right)\right), \\
P_{t+u_{n}}\left\{N_{n s}<C_{n}, U_{n s}\right\} & =P_{t+u_{n}}\left\{N_{n s \varepsilon}<C_{n}\right\} \exp \left\{-O\left(n u_{n}^{2} \omega\left(u_{n}\right)\right)\right\} \\
& =\Phi\left(C_{n}-E_{t+u_{n}}\left[N_{n s \varepsilon}\right]+O\left(n^{1 / 2} u_{n} \omega\left(u_{n}\right)\right)\right) .
\end{aligned}
$$

Из (2.24) u (2.25) следyет

$$
\begin{gathered}
\Phi^{-1}\left(P_{t}\left\{N_{n s \varepsilon}>C_{n}\right\}\right) \leqslant-C_{n}+E_{t}\left[N_{n s \varepsilon}\right]+O\left(n^{1 / 2} u_{n} \omega\left(u_{n}\right)\right), \\
\Phi^{-1}\left(P_{t+u_{n}}\left\{N_{n s \varepsilon}<C_{n}\right\}\right) \leqslant C_{n}-E_{t+u_{n}}\left[N_{n s \varepsilon}\right]+O\left(n^{1 / 2} u_{n} \omega\left(u_{n}\right)\right) .
\end{gathered}
$$

3 а м е ч а н и е 2.1. Неравенства (2.12)-(2.15) позволяют доказывать нижние границы для вероятностей умеренных уклонений без предположения существования экспоненциальных моментов у функции влияния $\varphi$. Результаты такого рода не являются принципиально новыми. Нижние границы для вероятностей умеренных уклонений сумм независимых одинаково распределенных случайных величин при предположениях подобного типа были доказаны А.Н. Архангельским [2]. Подобные результаты не означают, что нижние границы достигаются, хотя и показывают, что нормальная аппроксимация, по крайней мере как нижняя граница, без серьезных дополнительных предположений может быть продолжена на зону умеренных уклонений.

Как следует из оценок леммы 3.4, приведенной в следующем разделе,

$$
\begin{aligned}
E_{t}\left[N_{n 1}\right] & =-n^{1 / 2}\left(\rho_{n}+O\left(u_{n} \omega\left(u_{n}\right)\right)\right), \\
E_{t+u_{n}}\left[N_{n 1}\right] & =n^{1 / 2}\left(\rho_{n}+O\left(u_{n} \omega\left(u_{n}\right)\right)\right), \\
E_{t}\left[N_{n 1 \varepsilon}\right] & =-n^{1 / 2}\left(\rho_{n}+O\left(u_{n} \omega\left(u_{n}\right)\right)\right), \\
E_{t+u_{n}}\left[N_{n 1 \varepsilon}\right] & =n^{1 / 2}\left(\rho_{n}+O\left(u_{n} \omega\left(u_{n}\right)\right)\right), \\
E_{t}\left[N_{n 2 \varepsilon}\right] & =-\frac{1}{2} n^{1 / 2}\left(\rho_{n}+O\left(u_{n} \omega\left(u_{n}\right)\right)\right), \\
E_{t+u_{n}}\left[N_{n 2 \varepsilon}\right] & =\frac{3}{2} n^{1 / 2}\left(\rho_{n}+O\left(u_{n} \omega\left(u_{n}\right)\right)\right), \\
E_{t}\left[N_{n 3 \varepsilon}\right] & =O\left(n^{1 / 2} u_{n} \omega\left(u_{n}\right)\right), \\
E_{t+u_{n}}\left[N_{n 3 \varepsilon}\right] & =n^{1 / 2}\left(I^{1 / 2} u_{n}+O\left(u_{n} \omega\left(u_{n}\right)\right)\right) .
\end{aligned}
$$


2.2. MD и SMD эффективности в задачах проверки гипотез. Нижние границы для вероятностей умеренных уклонений ошибок первого и второго рода непосредственно вытекают из теоремы 2.1 и леммы Неймана-Пирсона.

Предположим, что необходимо проверить гипотезу $H_{t}: \theta=t$ против альтернатив $H_{t_{n}}: \theta=t_{n}=t+u_{n}$, где $u_{n} \rightarrow 0, n u_{n}^{2} \rightarrow \infty$ при $n \rightarrow \infty$. Для любого критерия $K_{n}$ обозначим его вероятности ошибок первого и второго рода через $\alpha\left(K_{n}\right)=\alpha\left(K_{n}, P_{t}\right)$ и $\beta\left(K_{n}\right)=\beta\left(K_{n}, P_{t_{n}}\right)$ соответственно. Определим последовательность критериев отношения правдоподобия $L_{n}=\chi\left(N_{n 1}-E_{t} N_{n 1}>C_{n}\right)$ и последовательность критериев $L_{n s}=\chi\left(N_{n s}-E_{t} N_{n s \varepsilon}>C_{n}\right)$, где $s=1,2,3$.

Теорема 2.2. Пусть статистический эксперимент Е имеет конечное информачионное количество Фишера в точке $t$, m.е. выполнено (2.1). Пусть $u_{n} \rightarrow 0, n u_{n}^{2} \rightarrow \infty n р и n \rightarrow \infty$. Тогда для любой последовательности критериев $K_{n}$ такой, что

$$
\left|\left(n^{1 / 2} u_{n} I^{1 / 2}\right)^{-1} \Phi^{-1}\left(\alpha\left(K_{n}\right)\right)\right|<C
$$

uлu

$$
\left|\left(n^{1 / 2} u_{n} I^{1 / 2}\right)^{-1} \Phi^{-1}\left(\beta\left(K_{n}\right)\right)\right|<C,
$$

uмeет место

$$
\liminf _{n \rightarrow \infty}\left(n^{1 / 2} u_{n} I^{1 / 2}\right)^{-1}\left(\Phi^{-1}\left(\alpha\left(K_{n}\right)\right)+\Phi^{-1}\left(\beta\left(K_{n}\right)\right)\right) \geqslant-1
$$

Если $K_{n}=L_{n}$ является последовательностью критериев отношения правдоподобия, удовлетворяющих условию

$$
-n^{1 / 2} u_{n} I^{1 / 2}<\Phi^{-1}\left(\alpha\left(L_{n}\right)\right)<C
$$

или

$$
-n^{1 / 2} u_{n} I^{1 / 2}<\Phi^{-1}\left(\beta\left(L_{n}\right)\right)<C
$$

то в (2.17) достигается равенство, т.е.

$$
\lim _{n \rightarrow \infty}\left(n^{1 / 2} u_{n} I^{1 / 2}\right)^{-1}\left(\Phi^{-1}\left(\alpha\left(L_{n}\right)\right)+\Phi^{-1}\left(\beta\left(L_{n}\right)\right)\right)=-1 .
$$

Из (2.17), (2.20) следует, что если $\alpha\left(K_{n}\right)<C<1$ u $\beta\left(K_{n}\right)<C<1$, mо

$$
\limsup _{n \rightarrow \infty}\left(n^{1 / 2} u_{n} I^{1 / 2}\right)^{-1}\left(\left|2 \ln \alpha\left(K_{n}\right)\right|^{1 / 2}+\left|2 \ln \beta\left(K_{n}\right)\right|^{1 / 2}\right) \leqslant 1,
$$

причем равенство достигается, если $K_{n}=L_{n}$.

Пусть вьполнено условие В. Тогда соотношения (2.17), (2.20), (2.21) будут справедливы, если в них и в (2.16), (2.18), (2.19) заменить $u_{n} I^{1 / 2}$ на $2 \rho_{n}$. 
Будем говорить, что последовательность критериев $K_{n}$, удовлетворяющая (2.16), является (MD) асимптотически эффективной в смысле вероятностей умеренных уклонений, если в (2.17) достигается равенство.

3 а м е ч а н и е 2.2. Аналогичное утверждение справедливо и для задачи проверки простой гипотезы против двусторонней альтернативы. Предположим, что необходимо проверить гипотезу $\theta=t$ против альтернатив $\theta=\theta_{n 1}=t+u_{n}$ или $\theta=\theta_{n 2}=t-u_{n}$. Для любого критерия $K_{n}$ обозначим $\beta\left(K_{n}\right)=\max \left\{\beta\left(K_{n}, P_{\theta_{n 1}}\right), \beta\left(K_{n}, P_{\theta_{n 1}}\right)\right\}$ и положим $\hat{\rho}_{n}=\min \left\{\rho\left(t, \theta_{n 1}\right), \rho\left(t, \theta_{n 2}\right)\right\}$. Тогда $(2.17),(2.20),(2.21)$ имеют место при тех же самых предположениях, если заменить $u_{n} I^{1 / 2}$ на $2 \widehat{\rho}_{n}$. Данное утверждение также является простым следствием теоремы 2.1 .

Д ок аз а тельст в о т е о ремы 2.2. Применяя лемму Неймана-Пирсона и используя неравенства $P_{t}\left\{N_{n 1}>C_{n}\right\}>P_{t}\left\{N_{n 1}>\right.$ $\left.C_{n}, U_{n 1}\right\}, P_{t+u_{n}}\left\{N_{n 1}<C_{n}\right\}>P_{t+u_{n}}\left\{N_{n 1}<C_{n}, U_{n 1}\right\}$ и (2.12)-(2.15), получаем (2.17) и (2.21). Доказательство (2.20) следует из (2.10) и (2.11), если ни одно из следующих соотношений не имеет места:

$$
\begin{aligned}
& \lim _{n \rightarrow \infty} \frac{1}{2 n^{1 / 2} \rho_{n}} \Phi^{-1}\left(\alpha\left(L_{n}\right)\right)=0, \\
& \lim _{n \rightarrow \infty} \frac{1}{2 n^{1 / 2} \rho_{n}} \Phi^{-1}\left(\alpha\left(L_{n}\right)\right)=-1, \\
& \lim _{n \rightarrow \infty} \frac{1}{2 n^{1 / 2} \rho_{n}} \Phi^{-1}\left(\beta\left(L_{n}\right)\right)=0, \\
& \lim _{n \rightarrow \infty} \frac{1}{2 n^{1 / 2} \rho_{n}} \Phi^{-1}\left(\beta\left(L_{n}\right)\right)=-1 .
\end{aligned}
$$

В этих случаях непосредственное применение $(2.10),(2.11)$ потребовало бы значения $\delta=0$. Предположим, что имеет место (2.22) (в остальных случаях рассуждения аналогичны). Если выполнено условие А или $\mathrm{B}$, то имеет место $\mathrm{C}$ при $\delta=0$. Следовательно, существует последовательность $\delta_{n}>0, \delta_{n} \rightarrow 0$ при $n \rightarrow \infty$, такая, что $(2.10),(2.11)$ имеют место при $\delta=\delta_{n}$ и $-\left(1-2 \delta_{n}\right) n^{1 / 2} \rho_{n}<C_{n}<\left(1-2 \delta_{n}\right) n^{1 / 2} \rho_{n}$. Поэтому можно определить последовательность критериев отношения правдоподобия $L_{n 1}$, удовлетворяющую $(2.20)$ и такую, что

$$
\lim _{n \rightarrow \infty}\left(n^{1 / 2} \rho_{n}\right)^{-1} \Phi^{-1}\left(\alpha\left(L_{n 1}\right)\right)=0
$$

и $\alpha\left(L_{n}\right)>\alpha\left(L_{n 1}\right), \beta\left(L_{n}\right)<\beta\left(L_{n 1}\right)$. Так как для $L_{n 1}$ справедливо $(2.20)$ и $L_{n}$ удовлетворяет $(2.17)$, это означает, что для $L_{n}$ также справедливо (2.20). Таким образом, (2.20) может быть доказано без условия С.

Теорема 2.3. Пусть выполнено условие В. Пусть $u_{n}>\mathrm{cn}^{-1 / 2}$, $n u_{n}^{2} \omega\left(u_{n}\right) \rightarrow 0$ при $n \rightarrow \infty$. Тогда для любой последовательности критериев $K_{n}$, удовлетворяющей (2.16), имеет место неравенство

$$
\liminf _{n \rightarrow \infty} n^{1 / 2} \rho_{n}\left(2 n^{1 / 2} \rho_{n}+\Phi^{-1}\left(\alpha\left(K_{n}\right)\right)+\Phi^{-1}\left(\beta\left(K_{n}\right)\right)\right) \geqslant 0 .
$$


Если условие С также выполнено и последовательность критериев отношения правдоподобия $L_{n}$ удовлетворяет неравенствам

$$
-2 n^{1 / 2} \rho_{n}\left(1+\delta_{1}\right)<\Phi^{-1}\left(\alpha\left(L_{n}\right)\right)<2 n^{1 / 2} \rho_{n} \delta_{1}
$$

นภน

$$
-2 n^{1 / 2} \rho_{n}\left(1+\delta_{1}\right)<\Phi^{-1}\left(\beta\left(L_{n}\right)\right)<2 n^{1 / 2} \rho_{n} \delta_{1}
$$

əде $\delta_{1}<2 \delta, m o$

$$
\lim _{n \rightarrow \infty} n^{1 / 2} \rho_{n}\left(2 n^{1 / 2} \rho_{n}+\Phi^{-1}\left(\alpha\left(L_{n}\right)\right)+\Phi^{-1}\left(\beta\left(L_{n}\right)\right)\right)=0 .
$$

Если выполнено условие А, то в (2.27)-(2.30) $\rho_{n}^{2}$ может быть заменено на $\frac{1}{4} I u_{n}^{2}$. В частности, это означает, что справедливо неравенство (1.6).

Если в (2.27) достигается равенство и

$$
-2 n^{1 / 2} \rho_{n}\left(1+\delta_{1}\right)<\Phi^{-1}\left(\alpha\left(K_{n}\right)\right)<2 n^{1 / 2} \rho_{n} \delta_{1}
$$

$\partial_{\Omega \Omega} \delta_{1}<2 \delta, m o$

$$
\begin{array}{r}
\lim _{n \rightarrow \infty} E_{t}\left\{\left|K_{n}-L_{n s}\right| \chi\left(U_{n s}\right)\right\} \alpha\left(K_{n}\right)^{-1}=0, \\
\lim _{n \rightarrow \infty} E_{t_{n}}\left\{\left|K_{n}-L_{n s}\right| \chi\left(U_{n s}\right)\right\} \beta\left(K_{n}\right)^{-1}=0, \\
\lim _{n \rightarrow \infty} E_{t}\left\{K_{n}\left(1-\chi\left(U_{n s}\right)\right)\right\} \alpha\left(K_{n}\right)^{-1}=0, \\
\lim _{n \rightarrow \infty} E_{t_{n}}\left\{\left(1-K_{n}\right)\left(1-\chi\left(U_{n s}\right)\right)\right\} \beta\left(K_{n}\right)^{-1}=0
\end{array}
$$

для $s=1,2$, если вьпполнень условия $\mathrm{B}, \mathrm{C}, u$ для $s=3$, если вьлолнень условия А, С. Здесь последовательности критериев $L_{n s}$ задань так, чmo $\alpha\left(L_{n s}\right)=\alpha\left(K_{n}\right) \partial \Omega s s=1,2,3$.

Назовем последовательность критериев $K_{n}$, удовлетворяющую условию (2.16), (SMD) сильно асимптотически эффективной в зоне умеренных уклонений, если в (2.27) достигается равенство.

Теорема 2.3 и приводимая ниже теорема 2.4 непосредственно следуют из теоремы 2.1 и леммы Неймана-Пирсона. Аналогичные рассуждения уже были приведены при доказательстве теоремы 2.2. Поэтому мы опустим эти доказательства.

3 а м е ч а н и е 2.3. Если $u_{n}=c n^{-1 / 2}$, то соотношения (2.27), (2.30) определяют нижнюю границу для эффективности по Питмену. Это утверждение не вытекает непосредственно из теоремы 2.1. Доказательства асимптотической нормальности логарифма отношения правдоподобия при справедливости гипотезы можно найти в монографиях [18], [22]. В случае последовательности альтернатив доказательство получается простой модификацией. Доказательство асимптотической нормальности статистик $N_{n 2}$ и $N_{n 3}$, по сушеству, основано на той же самой аналитике. 
3 а м е ч а н и е 2.4. Как следует из теоремы Гаека [11], если последовательность оценок $\hat{\theta}_{n}$ локально асимптотически минимаксна, то

$$
(n I)^{1 / 2}\left(\hat{\theta}_{n}-t\right)-2(n I)^{-1 / 2} \sum_{i=1}^{n} \varphi\left(X_{i}\right) \longrightarrow 0
$$

по вероятности при $n \rightarrow \infty$. Соотношения (2.31)-(2.34) могут рассматриваться как некоторый аналог утверждения Гаека для постановки задачи SMD эффективности.

Зададим функцию $s=\bar{\Phi}^{-1}(y)$ уравнением $y=2 \Phi(s)$.

Теорема 2.4. Пусть выполнено условие В. Пусть $u_{n}>\mathrm{Cn}^{-1 / 2}$, $n u_{n}^{2} \omega\left(u_{n}\right) \rightarrow 0$ при $n \rightarrow \infty$. Предположим, что стоит задача проверки гипотезь $H_{0}: \theta=t$ против альтернатив $H_{n}: \theta=\theta_{n 1}=t+u_{n}$ или $\theta=\theta_{n 2}=t-u_{n}$. Тогда для любой последовательности критериев $K_{n}$, удовлетворяющей (2.16), имеет место

$$
\begin{aligned}
\liminf _{n \rightarrow \infty}\left(4 n \widehat{\rho}_{n}^{2}\right)^{1 / 2}( & 4 n^{1 / 2} \widehat{\rho}_{n}+2 \bar{\Phi}^{-1}\left(\alpha\left(K_{n}\right)\right)+\Phi^{-1}\left(\beta\left(K_{n}, P_{n \theta_{n 1}}\right)\right) \\
& \left.+\Phi^{-1}\left(\beta\left(K_{n}, P_{n \theta_{n 2}}\right)\right)\right) \geqslant 0 .
\end{aligned}
$$

Здесь используются те же самье обозначения для $\hat{\rho}_{n}$ и $\beta\left(K_{n}\right)$, что ив замечании 2.2 .

Предположим, что выполнень условия В и С. Тогда для любой последовательности критериев отношения правдоподобия $L_{n}$, удовлетворяющей (2.28) или (2.29), нижняя гранииа в (2.35) достигается, т.е. левая часть (2.35) равна нулю.

Пусть выполнено условие А. Тогда в (2.35) можно положить $2 \widehat{\rho}_{n}=u_{n} I^{1 / 2}$.

Ясно, что соотношения, аналогичные (2.31)-(2.34), также могут быть доказаны и для постановки задачи теоремы 2.4 .

3 а м е ч а н и е 2.5. При доказательстве нижних границ в статистическом оценивании (см. доказательство теоремы 2.7) нами будет использован несколько более сильный аналог теоремы 2.3. Предположим, что стоит задача проверки гипотезы $H_{1 n}: \theta=t_{1 n}=t_{0}+v_{n}$ против альтернатив $H_{2 n}=t_{2 n}=t_{0}+v_{n}+u_{n}$, где $\left|v_{n}\right|<C u_{n}, u_{n}>C n^{-1 / 2}, n u_{n}^{2} \omega\left(u_{n}\right) \rightarrow 0$ при $n \rightarrow \infty$. Обозначим $\alpha\left(K_{n}\right)=\alpha\left(K_{n}, P_{t_{1 n}}\right), \beta\left(K_{n}\right)=\beta\left(K_{n}, P_{t_{2 n}}\right)$ и положим $\rho_{n}=\rho\left(P_{t_{1 n}}, P_{t_{2 n}}\right)$. Определим случайные величины $\xi_{n i}, \eta_{n i}$, $N_{n 1}, N_{n 2}, N_{n 1 \varepsilon}, N_{n 2 \varepsilon}$ и события $B_{n 1 i}, B_{n 2 i}, U_{n 1}, U_{n 2}$ так же, как и прежде, с единственным отличием, что параметр $t$ заменяется на $t_{n 1}$, т.е. $\xi_{n i}=$ $\ln \left[f\left(X_{i}, t_{n 2}\right) / f\left(X_{i}, t_{n 1}\right)\right], \eta_{n i}=\left(f\left(X_{i}, t_{n 2}\right) / f\left(X_{i}, t_{n 1}\right)\right)^{1 / 2}-1,1 \leqslant i \leqslant n$, и т.д. Тогда соотношения $(2.27),(2.30)-(2.34)$ теоремы 2.3 остаются справедливыми. Данное утверждение вытекает из того, что оценки в теореме 2.1 имеют равномерный характер. 


\subsection{MD и SMD эффективности в задачах оценивания.}

Впервые на возможность вывода нижних границ вероятностей больших уклонений в оценивании из аналогичных границ в проверке гипотез обратил внимание Р. Бахадур [3].

Теорема 2.5. Пусть выполнено условие В. Пусть $u_{n}>0, u_{n} \rightarrow 0$, $n u_{n}^{2} \rightarrow \infty$ nри $n \rightarrow \infty$. Тогда для любой последовательности оченок $\hat{\theta}_{n}$ параметра $\theta$

$$
\liminf _{n \rightarrow \infty} \max _{\theta=t, t+2 u_{n}} \frac{2}{n \rho^{2}\left(t, t+2 u_{n}\right)} \ln P_{\theta}\left\{\left|\hat{\theta}_{n}-\theta\right|>u_{n}\right\} \geqslant-1 .
$$

В частности, условие В выполнено, если в точке $t$ определено конечное информационное количество Фишера, т.е. имеет место (2.1). В этом случае в (2.36) можно положить $u_{n}^{2} I=\rho^{2}\left(t, t+2 u_{n}\right)$.

Легко видеть, что локально асимптотически минимаксная граница в своем традиционном виде является простым следствием теоремы 2.5 (см. [20]). Она приведена в следствии 2.1.

Следствие 2.1. Пусть в точке $t$ конечно информачионное количество Фишера, т.е. имеет место (2.1). Пусть $u_{n}>0, u_{n} \rightarrow 0, n u_{n}^{2} \rightarrow \infty$ при $n \rightarrow \infty$. Тогда для любой последовательности оченок $\hat{\theta}_{n}$

$$
\liminf _{\delta \rightarrow 0} \liminf _{n \rightarrow \infty} \sup _{|\theta-t|<\delta} \frac{2}{n u_{n}^{2} I} \ln P_{\theta}\left\{\left|\hat{\theta}_{n}-\theta\right|>u_{n}\right\} \geqslant-1 .
$$

Доказательство те о ремы 2.5. Рассмотрим задачу проверки гипотезы $\theta=t$ против альтернатив $\theta=t_{n}=t+2 u_{n}$. Используя оценки $\hat{\theta}_{n}=\hat{\theta}_{n}\left(X_{1}, \ldots, X_{n}\right)$ в качестве тестовых статистик, определим последовательность критериев $K_{n}=\chi\left(\hat{\theta}_{n}-t>u_{n}\right)$. Пусть $\alpha\left(K_{n}\right)=P_{t}\left\{\hat{\theta}_{n}-t>u_{n}\right\}<C<1$ и $\beta\left(K_{n}\right)=P_{t_{n}}\left\{\hat{\theta}_{n}-t_{n} \leqslant u_{n}\right\}<C<1$. Тогда, используя (2.21), имеем

$$
\begin{aligned}
& \left(2 n \rho^{2}\left(t, t_{n}\right)\right)^{-1 / 2}\left(\left|\ln P_{t}\left\{\hat{\theta}_{n}-t>u_{n}\right\}\right|^{1 / 2}+\left|\ln P_{t_{n}}\left\{\hat{\theta}_{n}-t_{n}<-u_{n}\right\}\right|^{1 / 2}\right) \\
& \quad \leqslant 1+o(1)
\end{aligned}
$$

при $n \rightarrow \infty$. Отсюда $P_{t}\left\{\hat{\theta}_{n}-t>u_{n}\right\} \leqslant P_{t}\left\{\left|\hat{\theta}_{n}-t\right|>u_{n}\right\}$ и $P_{t_{n}}\left\{\hat{\theta}_{n}-t<\right.$ $\left.u_{n}\right\}=P_{t_{n}}\left\{\hat{\theta}_{n}-t_{n}<-u_{n}\right\} \leqslant P_{t_{n}}\left\{\left|\hat{\theta}_{n}-t_{n}\right|>u_{n}\right\}$, что и влечет (2.36).

Назовем последовательность оценок $\hat{\theta}_{n} u_{n}$-состоятельной для значения параметра $\theta=t$, если для любого $\delta>0$ при всех $\theta,|\theta-t|<u_{n}$, имеет место $P_{\theta}\left\{\left|\hat{\theta}_{n}-\theta\right|>\delta u_{n}\right\} \rightarrow 0$ при $n \rightarrow \infty$.

Аналогично доказательству теоремы 2.5 доказывается и следующая теорема.

Теорема 2.6. Пусть выполнено условие В. Пусть $u_{n}>0, u_{n} \rightarrow$ $0, n u_{n}^{2} \rightarrow \infty$ при $n \rightarrow \infty$. Тогда если последовательность оченок $\hat{\theta}_{n}$ 
является $u_{n}$-состоятельной, то для любого $0<c<1$

$$
\begin{aligned}
\limsup _{n \rightarrow \infty}\left(2 n \rho_{n}^{2}\right)^{-1 / 2} & \left(\left|\ln P_{t}\left\{\left|\hat{\theta}_{n}-t\right|>c u_{n}\right\}\right|^{1 / 2}\right. \\
& \left.+\left|\ln P_{t+u_{n}}\left\{\left|\hat{\theta}_{n}-t-u_{n}\right|>(1-c) u_{n}\right\}\right|^{1 / 2}\right) \leqslant 1 .
\end{aligned}
$$

Для точной асимптотики вероятностей умеренных уклонений статистических оценок не удается получить нижних границ в столь же простой форме. Здесь задача также сводится к задачам проверки гипотез, однако в предлагаемом доказательстве их число растет с ростом объема выборки и, как следствие, супремум берется по целой окрестности точки $t$. Результаты, использующие расстояние Хеллингера, будут выражены в терминах функции $R(t, C, u)=\inf \{\rho(\theta, \theta+u):|\theta-t|<C u\}$. Значение функции $R(t, C, u)$ может рассматриваться как «наименее благоприятное значение» расстояния Хеллингера.

Теорема 2.7. Пусть выполнено условие А. Пусть $u_{n}>0$, $n u_{n}^{2} \omega\left(u_{n}\right) \rightarrow 0, n u_{n}^{2} \rightarrow \infty$ nри $n \rightarrow \infty$. Тогда для любой последовательности оченок $\hat{\theta}_{n}$

$$
\liminf _{n \rightarrow \infty} \sup _{|\theta-t|<C_{n} u_{n}} \frac{P_{\theta}\left\{\left|\hat{\theta}_{n}-\theta\right|>u_{n}\right\}}{2 \Phi\left(-(n I)^{1 / 2} u_{n}\right)} \geqslant 1
$$

для всякой последовательности $C_{n} \rightarrow \infty$ при $n \rightarrow \infty$.

Если в (2.39) достигается равенство, т.е.

$$
\lim _{n \rightarrow \infty} \sup _{|\theta-t|<C_{n} u_{n}} \frac{P_{\theta}\left\{\left|\hat{\theta}_{n}-\theta\right|>u_{n}\right\}}{2 \Phi\left(-(n I)^{1 / 2} u_{n}\right)}=1,
$$

то для любой последовательности $\theta_{n},\left|\theta_{n}-t\right|<C_{n} u_{n}, C_{n} \rightarrow \infty$, $n C_{n}^{2} u_{n}^{2} \omega\left(C_{n} u_{n}\right) \rightarrow 0$ при $n \rightarrow \infty$ справедливь следуюшие утверждения.

(i) Для последовательности событий $V_{3 n}=\bigcap_{i=1}^{n}\left\{X_{i}:\left|\varphi\left(X_{i}\right)\right|<\right.$ $\left.\varepsilon u_{n}^{-1}\right\}, 0<\varepsilon<\frac{1}{3}$, uмеет место $P_{\theta_{n}}\left(V_{3 n}\right) \rightarrow 1$ npu $n \rightarrow \infty u$

$$
\begin{aligned}
& \lim _{n \rightarrow \infty} \frac{1}{\Phi\left(-(n I)^{1 / 2} u_{n}\right)} E_{\theta_{n}}\left\{\mid \chi\left(\left|\hat{\theta}_{n}-\theta_{n}\right|>u_{n}\right)\right. \\
&\left.-\chi\left(\left|\frac{2}{n I} \sum_{i=1}^{n} \tau_{i}-\theta_{n}\right|>u_{n}\right) \mid \chi\left(V_{3 n}\right)\right\}=0=0 . \\
& \lim _{n \rightarrow \infty} \frac{1}{\Phi\left(-(n I)^{1 / 2} u_{n}\right)} E_{\theta_{n}}\left[\chi\left(\left|\hat{\theta}_{n}-\theta_{n}\right|>u_{n}\right)\left(1-\chi\left(V_{3 n}\right)\right)\right]=0 .
\end{aligned}
$$

(ii) Для последовательности событий

$$
V_{1 n}=\bigcap_{i=1}^{n}\left\{X_{i}:\left|\xi_{n i}\left(\theta_{n}\right)\right|<\varepsilon\right\}, \quad 0<\varepsilon<\frac{1}{3},
$$


uмеет место $P_{t}\left(V_{1 n}\right) \rightarrow 1$ npu $n \rightarrow \infty u$

$$
\begin{aligned}
\lim _{n \rightarrow \infty} \frac{1}{\Phi\left(-(n I)^{1 / 2} u_{n}\right)} E_{\theta_{n}}\left\{\mid \chi\left(\left|\hat{\theta}_{n}-\theta_{n}\right|>u_{n}\right)\right. \\
\left.-\chi\left(\left|\sum_{i=1}^{n} \xi_{n i}\left(\theta_{n}\right)+\frac{1}{2} n I u_{n}^{2}\right|>n u_{n}^{2} I\right) \mid \chi\left(V_{1 n}\right)\right\}=0, \\
\lim _{n \rightarrow \infty} \frac{1}{\Phi\left(-(n I)^{1 / 2} u_{n}\right)} E_{\theta_{n}}\left[\chi\left(\left|\hat{\theta}_{n}-\theta_{n}\right|>u_{n}\right)\left(1-\chi\left(V_{1 n}\right)\right)\right]=0 .
\end{aligned}
$$

(iii) Для последовательности собьтий $V_{2 n}=\bigcap_{i=1}^{n}\left\{X_{i}:\left|g\left(X_{i}, \theta_{n}\right)\right|<\right.$ ह\} имеет место $P_{\theta_{n}}\left(V_{2 n}\right) \rightarrow 0$ npu $n \rightarrow \infty u$

$$
\begin{aligned}
& \lim _{n \rightarrow \infty} \frac{1}{\Phi\left(-(n I)^{1 / 2} u_{n}\right)} E_{\theta_{n}}\left\{\mid \chi\left(\left|\hat{\theta}_{n}-\theta_{n}\right|>u_{n}\right)\right. \\
&\left.-\chi\left(\left|\sum_{i=1}^{n} \eta_{n i}\left(\theta_{n}\right)+\frac{1}{8} n I u_{n}^{2}\right|>\frac{1}{2} n u_{n}^{2} I\right) \mid \chi\left(V_{2 n}\right)\right\}=0 \\
& \lim _{n \rightarrow \infty} \frac{1}{\Phi\left(u_{n}(n I)^{1 / 2}\right)} E_{\theta_{n}}\left[\chi\left(\left|\hat{\theta}_{n}-\theta_{n}\right|>u_{n}\right)\left(1-\chi\left(V_{2 n}\right)\right)\right]=0 .
\end{aligned}
$$

Пусть выполнено условие В. Тогда для любой последовательности оценок $\hat{\theta}_{n}$ имеет место неравенство

$$
\liminf _{n \rightarrow \infty} \sup _{|\theta-t|<C_{n} u_{n}} \frac{P_{\theta}\left\{\left|\hat{\theta}_{n}-\theta\right|>u_{n}\right\}}{2 \Phi\left(-2 n^{1 / 2} R\left(t, C_{n}, u_{n}\right)\right)} \geqslant 1
$$

для любой последовательности $C_{n} \rightarrow \infty$ nри $n \rightarrow \infty$.

Мы скажем, что последовательность оценок $\hat{\theta}_{n}$ является MD (соответственно SMD) асимптотически эффективной в смысле вероятностей умеренных уклонений (соответственно точной асимптотики вероятностей умеренных уклонений), если достигается равенство в (2.36) (соответственно в (2.39)).

3 а м е ч а н и е 2.6. Теоремы 2.1-2.7 легко обобщаются на случай $k$ выборок. Пусть $X_{j 1}, \ldots, X_{j n_{j}}, 1 \leqslant j \leqslant k$, - независимые одинаково распределенные случайные величины, имеющие вероятностные меры $P_{j \theta}$, $\theta \in \mathbf{R}^{1}, n=n_{1}+\cdots+n_{k}$, и пусть $n_{j} / n \rightarrow \nu_{j}$ при $n \rightarrow \infty$. Предположим, что вероятностные меры $P_{j \theta}, 1 \leqslant j \leqslant k$, удовлетворяют условию А. Тогда выполнены соотношения $(2.17),(2.20),(2.21),(2.26),(2.38)$, причем $\rho_{n}$ задается равенством

$$
\rho_{n}^{2}=\sum_{j=1}^{k} \nu_{j} \rho^{2}\left(P_{j t_{n 1}}, P_{j t_{n 2}}\right) .
$$

Предположим, что вероятностные меры $P_{j \theta}, 1 \leqslant j \leqslant k$, удовлетворяют условию А, и обозначим $I_{j}=I_{j t}$ информационное количество Фишера $P_{j \theta}$ 
в точке $\theta=t$. Предположим, что $\nu_{j}, 1 \leqslant j \leqslant k$, удовлетворяют следующему условию.

D. $u_{n}^{2} \max \left\{\left|n_{j}-n \nu_{j}\right|: 1 \leqslant j \leqslant k\right\} \rightarrow 0$ при $n \rightarrow \infty$.

При этом дополнительном условии соотношения $(2.27),(2.30)-(2.34)$, (2.39) также выполнены, причем $I=\sum_{j=1}^{k} \nu_{j} I_{j}$.

3 а м е ч а н и е 2.7. Обозначим $\Xi\left(P_{t}\right)$ множество всех отображений $\Xi: u \rightarrow P_{t+u}, u \in(-\delta, \delta), \delta>0$, удовлетворяющих $(2.5)-(2.7)$ для фиксированных постоянных $C, C_{\varepsilon}$. Тогда из доказательств теорем 2.1-2.7 легко видеть, что нижние границы $(2.17),(2.21),(2.27)$, $(2.35),(2.36),(2.38),(2.39),(2.46)$ являются равномерными относительно $\Psi \in \Xi\left(P_{t}\right)$. Иначе говоря, если мы поместим inf $\Psi \in \Xi\left(P_{t}\right)$ после $\liminf _{n \rightarrow \infty}$ в $(2.17),(2.27),(2.35),(2.36),(2.39),(2.46)$ и $\sup _{\Psi \in \Xi\left(P_{t}\right)}$ после $\limsup _{n \rightarrow \infty}$ в $(2.21),(2.38)$. Легко видеть, что сходимость в $(2.20),(2.30)$ является также равномерной относительно $\Psi \in \Xi\left(P_{t}\right)$.

Доказательство теоремы 2.7 основано на следующей лемме.

Лемма 2.1. Пусть выполнено условие В. Предположим, ито необходимо проверить гипотезу $H_{0}: \theta=t_{n}$ против альтернативь $H_{1}: \theta=t_{n}+2 u_{n}$, где $\left|t_{n}-t\right|<C_{n} u_{n}, n C_{n}^{2} u_{n}^{2} \omega\left(C_{n} u_{n}\right) \rightarrow 0$ npu $n \rightarrow \infty$. Тогда для любой последовательности критериев $K_{n}$ такой, что

$$
\alpha\left(K_{n}\right) \leqslant 2 \Phi\left(-2 n^{1 / 2} \rho_{n}\right) \quad \text { u } \beta\left(K_{n}\right) \leqslant 2 \Phi\left(-2 n^{1 / 2} \rho_{n}\right)
$$

имеет место неравенство

$$
\liminf _{n \rightarrow \infty} \frac{\alpha\left(K_{n}\right) \beta\left(K_{n}\right)}{\Phi^{2}\left(-2 n^{1 / 2} \rho_{n}\right)} \geqslant 1 .
$$

Доказательство лем мы 2.1. Так как

$$
\exp \left\{-\frac{\left(x-x^{-1} \ln c\right)^{2}}{2}\right\}=c \exp \left\{-\frac{x^{2}}{2}\right\}(1+o(1)) \quad \text { при } \quad x \rightarrow-\infty,
$$

получаем

$$
\Phi^{-1}(c \Phi(x))=x-x^{-1} \ln c(1+o(1)) \quad \text { при } \quad x \rightarrow-\infty
$$

И

$$
\Phi\left(x+x^{-1} \ln c\right)=c^{-1} \Phi(x)(1+o(1)) \quad \text { при } \quad x \rightarrow-\infty .
$$

Из (2.27), используя $(2.47),(2.49),(2.50)$, получаем

$$
\begin{aligned}
\alpha\left(K_{n}\right) & \geqslant \Phi\left(-4 n^{1 / 2} \rho_{n}-\Phi^{-1}\left(\beta\left(K_{n}\right)\right)\right)(1+o(1)) \\
& \geqslant \Phi\left(-4 n^{1 / 2} \rho_{n}-\Phi^{-1}\left(2 \Phi\left(-2 n^{1 / 2} \rho_{n}\right)\right)\right)(1+o(1)) \\
& \geqslant \frac{1}{2} \Phi\left(-2 n^{1 / 2} \rho_{n}\right)(1+o(1)) .
\end{aligned}
$$

Рассуждая аналогично, имеем

$$
\beta\left(K_{n}\right) \geqslant \frac{1}{2} \Phi\left(-2 n^{1 / 2} \rho_{n}\right)(1+o(1)) .
$$


Пусть $\beta\left(K_{n}\right)=c_{n} \Phi\left(-2 n^{1 / 2} \rho_{n}\right)$ и $\frac{1}{2} \leqslant c_{n} \leqslant 2$. Тогда, в силу $(2.27),(2.49)$, $(2.50)$, получаем

$$
\begin{aligned}
\alpha\left(K_{n}\right) & \geqslant \Phi\left(-4 n^{1 / 2} \rho_{n}-\Phi^{-1}\left(c_{n} \Phi\left(-2 n^{1 / 2} \rho_{n}\right)\right)\right)(1+o(1)) \\
& =\Phi\left(-2 n^{1 / 2} \rho_{n}-\frac{\ln c_{n}}{2 n^{1 / 2} \rho_{n}}(1+o(1))\right) \\
& =c_{n}^{-1} \Phi\left(-2 n^{1 / 2} \rho_{n}\right)(1+o(1)) .
\end{aligned}
$$

Отсюда и следует (2.48).

Д ок азатель с т о т е о рем ы 2.7. Доказательство будет проведено, если выполнено условие А. В случае выполнения условия В отличия незначительны.

Для произвольной последовательности значений параметра $t_{n}$, $\left|t_{n}-t\right|<C_{n} u_{n}, C_{n} \rightarrow \infty, n C_{n}^{2} u_{n}^{2} \omega\left(C_{n} u_{n}\right) \rightarrow 0$ при $n \rightarrow \infty$, рассмотрим $2 l$ задач проверки гипотез $H_{n j}: \theta=t_{n j}=t_{n}+2 j u_{n}$ против альтернатив $H_{n, j+1}: \theta=t_{n, j+1}, m \doteq-l \leqslant j \leqslant l-1$. Определим последовательности критериев $K_{n j}=\chi\left(\hat{\theta}_{n}-t_{n j}>u_{n}\right)$. Обозначим $\alpha_{n j}, \beta_{n j}$ соответственно вероятности ошибок первого и второго рода критериев $K_{n j}, m \leqslant j \leqslant l-1$. Положим $r_{n j}=\alpha_{n j} / \Phi\left(-(n I)^{1 / 2} u_{n}\right)-1, s_{n j}=1-\beta_{n j} / \Phi\left(-(n I)^{1 / 2} u_{n}\right)$.

Предположим противное, что (2.39) не имеет места. Это означает, что существуют последовательность $t_{n},\left|t_{n}-t\right|<C_{n} u_{n}$, и $\varepsilon>0$ такие, что

$$
\begin{gathered}
r_{n, 1}-s_{n, 0}<-\varepsilon \quad \text { и } \quad r_{n, j}-s_{n, j-1}<o(1) \\
\text { для всех } m+1 \leqslant j \leqslant l-1 .
\end{gathered}
$$

Заметим, что $r_{n m}<1+o(1)$, так как если $r_{n m}>1+\delta$ и $\delta>0$, то

$$
\begin{aligned}
P_{t_{n m}}\left\{\left|\hat{\theta}_{n}-t_{n m}\right|>u_{n}\right\} & \geqslant P_{t_{n m}}\left\{\hat{\theta}_{n}-t_{n m}>u_{n}\right\} \\
& \geqslant(2+\delta) \Phi\left(-(n I)^{1 / 2} u_{n}\right),
\end{aligned}
$$

что означает (2.39). Аналогично получаем $s_{n l}>-1+o(1)$.

Из леммы 2.1 следует, что

$$
\left(1+r_{n j}\right)\left(1-s_{n j}\right)>1+o(1) \quad \text { для всех } m+1 \leqslant j \leqslant l-1 .
$$

Используя соотношения $r_{n m}<1+o(1)$ и $(2.53),(2.51)$, по индукции получаем $s_{n m}<\frac{1}{2}+o(1), r_{n, m+1}<\frac{1}{2}+o(1), s_{n, m+1}<\frac{1}{3}+o(1), \ldots, s_{n, m+j}<$ $1 /(j+2)+o(1), r_{n, m+j+1}<1 /(j+2)+o(1)$ и т.д. Аналогично, используя $(2.51), s_{n l}>-1+o(1)$ и $(2.53)$, получаем $r_{n, l-j}>-(j+2)^{-1}+o(1)$, $s_{n, l-j}>-(j+2)^{-1}+o(1)$ для $1 \leqslant j<l$. Так как $o(1)$ во всех оценках не зависит от $j$, данные неравенства противоречат $r_{n, 1}-s_{n, 0}<-\varepsilon$ при $l \rightarrow \infty$.

Предположим, что в (2.39) имеет место равенство. Пусть $l=l_{n}=$ $o\left(C_{n}\right), l_{n} \rightarrow \infty$ при $n \rightarrow \infty$. Тогда, рассуждая аналогично, получаем $s_{n, l-j}>-1 / j+o(1), r_{n, l-j}>-1 / j+o(1), s_{n, m+j}<1 / j+o(1), r_{n, m+j}<$ 
$1 / j+o(1)$ при $n \rightarrow \infty$. Следовательно, в задачах проверки гипотез $H_{n, 0}: \theta=t_{n}$ против $H_{n, 1}: \theta=t_{n, 1}$ и $H_{n, 0}: \theta=t_{n}$ против $H_{n,-1}: \theta=t_{n,-1}$ в (2.27) должно достигаться равенство. В силу (2.31)-(2.34) это означает (2.40)-(2.45) для $\theta_{n}=t_{n}$. Доказательство теоремы 2.7 завершено.

\section{3. Доказательство теоремы 2.1}

Доказательство теоремы 2.1 основано на одном из вариантов теоремы о вероятностях больших уклонений для схемы серий независимых случайных величин (см. [10]) и следующем представлении отношения правдоподобия:

$$
\begin{aligned}
& \prod_{i=1}^{n} \frac{f\left(X_{i}, t+u_{n}+v_{n}\right)}{f\left(X_{i}, t+v_{n}\right)} \\
& \quad=\exp \left\{2 \sum_{i=1}^{n}\left(\eta_{n i}\left(t+v_{n}, u_{n}, X_{i}\right)+\frac{1}{2} \rho_{n}^{2}\right)-2 n \rho_{n}^{2}+\zeta_{n 1}\right\},
\end{aligned}
$$

где $\zeta_{n 1} \rightarrow 0$ по вероятности при $n \rightarrow \infty$.

По сравнению с локальной асимптотической нормальностью представление (3.1) приводит к несколько более точным результатам. Оценки базируются на стандартной технике, развитой для доказательства асимптотической нормальности логарифма отношения правдоподобия. Необходимо только получить для них нужный вид остаточных членов.

Чтобы упростить обозначения, предположим, что $t=0$, и будем обозначать $E \zeta=E_{t} \zeta, P(D)=P_{t}(D)$ для любой случайной величины $\zeta$ и произвольного события $D$. Определим события $A_{1 n}=A_{1 n}(\varepsilon)=$ $\left\{x_{1}:\left|\xi_{n}\left(x_{1}\right)\right|>\varepsilon, x_{1} \in S\right\}, A_{2 n}=A_{2 n}(\varepsilon)=\left\{x_{1}:\left|\eta_{n}\left(x_{1}\right)\right|>\varepsilon, x_{1} \in S\right\}$ и $A_{3 n}=A_{3 n}(\varepsilon)=\left\{x_{1}:\left|\varphi\left(x_{1}\right)\right|>\varepsilon u_{n}^{-1}, x_{1} \in S\right\}$, где $\varepsilon>0$.

Мы начнем с доказательства вспомогательных лемм.

Лемма 3.1. Из условия А следует выполнение условия В.

Д ок а з а т ел ь с т в о. Так как $2 E \eta_{n}^{2}>u_{n}^{2} E \tau^{2}-2 E\left(\eta_{n}-u_{n} \tau\right)^{2}$, то (2.6) следует из (2.2).

В силу неравенства Чебышева, используя (2.4), получаем

$$
\begin{gathered}
E\left[\tau^{2} \chi\left(A_{3 n}\left(\frac{\varepsilon}{2}\right)\right)\right]<C \omega\left(\frac{2 u_{n}}{\varepsilon}\right), \\
E\left[|\tau|^{3} \chi\left(B_{3 n}\right)\right]<C u_{n}^{-1} \omega\left(u_{n}\right) E\left[\tau^{2} \omega^{-1}\left(\tau^{-1}\right)\right]<C \frac{\omega\left(u_{n}\right)}{u_{n}}, \\
P\left(A_{3 n}\right)<C u_{n}^{2} E\left[|\tau|^{2} \chi\left(A_{3 n}\right)\right]<C u_{n}^{2} \omega\left(u_{n}\right) .
\end{gathered}
$$

Применяя неравенство Чебышева и (2.2), (3.2), получаем

$$
E\left[\eta_{n}^{2} \chi\left(A_{2 n}\right)\right]<3 E\left(\eta_{n}-u_{n} \tau\right)^{2}+3 u_{n}^{2} E\left[\tau^{2} \chi\left\{\left|\eta_{n}-u_{n} \tau\right|>\frac{\varepsilon}{2} \text { и } u_{n}|\tau|<\frac{\varepsilon}{2}\right\}\right]
$$




$$
\begin{aligned}
& +3 u_{n}^{2} E\left[\tau^{2} \chi\left(A_{3 n}\left(\frac{\varepsilon}{2}\right)\right)\right] \leqslant C E\left(\eta_{n}-u_{n} \tau\right)^{2}+C P\left\{\left|\eta_{n}-u_{n} \tau\right|>\frac{\varepsilon}{2}\right\} \\
& +C u_{n}^{2} \omega\left(u_{n}\right)<C E\left(\eta_{n}-u_{n} \tau\right)^{2}+C u_{n}^{2} \omega\left(u_{n}\right)<C u_{n}^{2} \omega\left(u_{n}\right) .
\end{aligned}
$$

Используя (2.2), (3.3), (3.4), получаем

$$
\begin{aligned}
& E\left[\left|\eta_{n}\right|^{3} \chi\left(B_{2 n}\right)\right]<8 E\left[\left|\eta_{n}-u_{n} \tau\right|^{3} \chi\left(\left|\eta_{n}-u_{n} \tau\right|<2 \varepsilon\right)\right] \\
& \quad+8 u_{n}^{3} E\left[|\tau|^{3} \chi\left(B_{3 n}\right)\right]+8 E\left[\left|\eta_{n}\right|^{3} \chi\left(\left|\eta_{n}\right|<\varepsilon, u_{n}|\tau|>\varepsilon\right)\right] \\
& \quad<C E\left|\eta_{n}-u_{n} \tau\right|^{2}+C u_{n}^{3} E\left[|\tau|^{3} \chi\left(B_{3 n}\right)\right]+C \varepsilon^{3} P\left(A_{3 n}\right)<C u_{n}^{2} \omega\left(u_{n}\right)
\end{aligned}
$$

Это завершает доказательство леммы 3.1 .

Обозначим $\eta_{v}(u)=f^{1 / 2}\left(X_{1}, v+u\right) f^{-1 / 2}\left(X_{1}, v\right)-1, \eta(v+u)=\eta_{0}(v+u)$ и $\eta_{u}=\eta_{u}(-u)=f^{1 / 2}\left(X_{1}, 0\right) f^{-1 / 2}\left(X_{1}, u\right)-1$ для всех $u, v \in \mathbf{R}^{1}$.

Следующая лемма показывает, что аналог условия В имеет место и для вероятностных мер $P_{u_{n}}$. Как следствие, соотношения $(2.25)$ и $(2.27)$ для $s=1,2$ будут вытекать из (2.24) и (2.26).

Лемма 3.2. Пусть выполнено условие В. Тогда для всех и из некоторой окрестности $t=0$

$$
\begin{aligned}
& E_{u}\left[\eta_{u}^{2} \chi\left(\left|\eta_{u}\right|>\varepsilon\right)\right]<C u^{2} \omega(u), \\
& E_{u}\left[\eta_{u}^{2} \chi\left(\left|\eta_{u}\right|<\varepsilon\right)\right]>C u^{2}, \\
& E_{u}\left[\left|\eta_{u}\right|^{3} \chi\left(\left|\eta_{u}\right|<\varepsilon\right)\right]<C u^{2} \omega(u) .
\end{aligned}
$$

Доказательство (3.7)-(3.9) следует из того, что $\eta_{u_{n}}=-\eta_{n} /\left(\eta_{n}+1\right)$ и $P_{t+u, t}^{s}(S)=O\left(u^{2} \omega(u)\right), P_{t, t-\uparrow}^{s}(S)=O\left(u^{2} \omega(u)\right)$. Данные оценки будут опущены.

Обозначим $\tau_{u}=\varphi\left(X_{1}\right) \chi\left(u\left|\varphi\left(X_{1}\right)\right|<\varepsilon\right)$.

Лемма 3.3. Пусть вьполнено условие $\mathrm{A}, u$ пусть $C_{n} \rightarrow \infty$, $n C_{n}^{2} u_{n}^{2} \omega\left(C_{n} u_{n}\right) \rightarrow 0$ при $n \rightarrow \infty$. Тогда для любого $\left|v_{n}\right|<C_{n} u_{n}$

$$
\int\left(g\left(x, v_{n}, u_{n}\right)-u_{n} \varphi(x) \chi\left(|\varphi(x)|<\varepsilon u_{n}^{-1}\right)\right)^{2} d P_{v_{n}}<C_{n}^{2} u_{n}^{2} \omega\left(C_{n} u_{n}\right) .
$$

Д о к а з а т ел ь с т в о. В силу (2.2) имеем

$$
\begin{aligned}
E_{v_{n}}( & \left.\eta_{v_{n}}\left(u_{n}\right)-u_{n} \tau_{u_{n}}\right)^{2} \\
\leqslant & E\left(\eta\left(v_{n}+u_{n}\right)-\eta\left(v_{n}\right)-u_{n} \tau_{u_{n}}\left(1+\eta\left(v_{n}\right)\right)^{2}\right)^{2}+C P_{v_{n}, 0}^{s}(S) \\
\leqslant & 8 E\left(\eta\left(v_{n}+u_{n}\right)-\left(v_{n}+u_{n}\right) \tau_{u_{n}}\right)^{2}+8 E\left(\eta\left(v_{n}\right)-v_{n} \tau_{u_{n}}\right)^{2} \\
& +8 u_{n}^{2} E \tau_{u_{n}}^{2}\left(\eta\left(v_{n}\right)-v_{n} \tau_{u_{n}}\right)^{2}+8 u_{n}^{2} v_{n}^{2} E \tau_{u_{n}}^{4}+C P_{v_{n} 0}^{s}(S) \\
\leqslant & C C_{n}^{2} u_{n}^{2} \omega\left(C_{n} u_{n}\right)
\end{aligned}
$$


так как по $(2.2),(3.4)$ и $(3.5)$

$$
\begin{aligned}
E\left(\eta\left(v_{n}\right)-v_{n} \tau_{u_{n}}\right)^{2} \leqslant & E\left[\left(\eta\left(v_{n}\right)-v_{n} \tau\right)^{2} \chi\left(B_{3 n}\right)\right] \\
& +E\left[\eta^{2}\left(v_{n}\right) \chi\left(A_{3 n} \cap B_{2 n}\left(v_{n}\right)\right)\right]+E\left[\eta^{2}\left(v_{n}\right) \chi\left(A_{2 n}\left(v_{n}\right)\right)\right] \\
\leqslant & C v_{n}^{2} \omega\left(v_{n}\right)+\varepsilon^{2} P\left(A_{3 n}\right)<C v_{n}^{2} \omega\left(v_{n}\right),
\end{aligned}
$$

где $B_{2 n}\left(v_{n}\right)=\left\{X_{1}:\left|\bar{\eta}\left(v_{n}\right)\right|<\varepsilon\right\}, A_{2 n}\left(v_{n}\right)=\left\{X_{1}:\left|\bar{\eta}\left(v_{n}\right)\right|>\varepsilon\right\}$. Лемма 3.3 доказана.

3 а м е ч а и и е 3.1. Лемма 3.3 позволяет доказать аналог теоремы 2.3 в постановке задачи замечания 2.5. Легко видеть, что (2.4), (3.10) влекут (2.5)-(2.7) для $t=t_{0}+v_{n}$. Для доказательства этого достаточно взять в (3.5), (3.6) математическое ожидание относительно $v_{n}$ и заменить случайные величины $\eta_{n}$ и $\tau$ случайными величинами $\eta_{v_{n}}\left(v_{n}+u_{n}\right)$ и $\tau_{u_{n}}$ соответственно. Следовательно, из леммы 3.3 следует, что (2.24)-(2.27) при $s=1,2,3$ справедливы для $t=t_{0}+v_{n}$, $\left|v_{n}\right|<C_{n} u_{n}$. Таким образом, замечание 2.5 доказано, если выполнено условие А.

Следующая лемма содержит оценки остаточных членов в асимптотике первых двух моментов $\xi_{n}, \eta_{n}$ и $\tau$.

Лемма 3.4. Пусть выполнено условие В. Пусть $0<\varepsilon<\frac{1}{16}, u$ пусть $u_{n} \rightarrow 0, n u_{n}^{2} \rightarrow \infty$ nрu $n \rightarrow \infty$. Тогда

$$
\begin{aligned}
E_{t}\left[\xi_{n} \chi\left(B_{1 n}\right)\right] & =-2 \rho_{n}^{2}+O\left(u_{n}^{2} \omega\left(u_{n}\right)\right), \\
E_{t+u_{n}}\left[\xi_{n} \chi\left(B_{1 n}\right)\right] & =2 \rho_{n}^{2}+O\left(u_{n}^{2} \omega\left(u_{n}\right)\right), \\
E_{t}\left[\xi_{n}^{2} \chi\left(B_{1 n}\right)\right] & =E_{t+u_{n}}\left[\xi_{n}^{2} \chi\left(B_{1 n}\right)\right]+O\left(u_{n}^{2} \omega\left(u_{n}\right)\right) \\
& =4 \rho_{n}^{2}+O\left(u_{n}^{2} \omega\left(u_{n}\right)\right), \\
E_{t}\left[\eta_{n} \chi\left(B_{2 n}\right)\right] & =E_{t} \eta_{n}+O\left(u_{n}^{2} \omega\left(u_{n}\right)\right)=-\frac{1}{2} \rho_{n}^{2}+O\left(u_{n}^{2} \omega\left(u_{n}\right)\right), \\
E_{t+u_{n}}\left[\eta_{n} \chi\left(B_{2 n}\right)\right] & =E_{t+u_{n}}\left[\eta_{n}\right]+O\left(u_{n}^{2} \omega\left(u_{n}\right)\right)=\frac{3}{2} \rho_{n}^{2}+O\left(u_{n}^{2} \omega\left(u_{n}\right)\right), \\
E_{t}\left[\eta_{n}^{2}\right] & =E_{t}\left[\eta_{n}^{2} \chi\left(B_{2 n}\right)\right]+O\left(u_{n}^{2} \omega\left(u_{n}\right)\right) \\
& =E_{t+u_{n}}\left[\eta_{n}^{2} \chi\left(B_{2 n}\right)\right]+O\left(u_{n}^{2} \omega\left(u_{n}\right)\right) \\
& =\rho_{n}^{2}+O\left(u_{n}^{2} \omega\left(u_{n}\right)\right) .
\end{aligned}
$$

Пусть выполнень условия В $и$ С. Тогда

$$
\begin{aligned}
E_{t}\left[\xi_{n}\right] & =-2 \rho_{n}^{2}+O\left(u_{n}^{2} \omega\left(u_{n}\right)\right), \\
E_{t+u_{n}}\left[\xi_{n}\right] & =2 \rho_{n}^{2}+O\left(u_{n}^{2} \omega\left(u_{n}\right)\right), \\
E_{t}\left[\xi_{n}^{2}\right] & =E_{t+u_{n}}\left[\xi_{n}^{2}\right]+O\left(u_{n}^{2} \omega\left(u_{n}\right)\right)=4 \rho_{n}^{2}+O\left(u_{n}^{2} \omega\left(u_{n}\right)\right) .
\end{aligned}
$$

Если выполнено условие A, то $\rho_{n}^{2}=\frac{1}{4} I u_{n}^{2}+O\left(u_{n}^{2} \omega\left(u_{n}\right)\right) u$

$$
E_{t}\left[\tau \chi\left(B_{3 n}\right)\right]=O\left(u_{n} \omega\left(u_{n}\right)\right)
$$




$$
\begin{aligned}
E_{t+u_{n}}\left[\tau \chi\left(B_{3 n}\right)\right] & =\frac{1}{2} I u_{n}+O\left(u_{n} \omega\left(u_{n}\right)\right) \\
E_{t}\left[\tau^{2} \chi\left(B_{3 n}\right)\right] & =E_{t+u_{n}}\left[\tau^{2} \chi\left(B_{3 n}\right)\right]+O\left(\omega\left(u_{n}\right)\right)=\frac{1}{4} I+O\left(\omega\left(u_{n}\right)\right)
\end{aligned}
$$

Д ок аза те льст в о. Так как $\left|\xi_{n}\right|>\varepsilon, 0<\varepsilon<\frac{1}{4}$ означает $\left|\eta_{n}\right|>\frac{\varepsilon}{4}$, то в силу $(2.5),(2.9)$

$$
\begin{aligned}
& P\left(A_{1 n}\right)<P\left(A_{2 n}\left(\frac{\varepsilon}{4}\right)\right) \leqslant 16 \varepsilon^{-2} E\left[\eta_{n}^{2} \chi\left(A_{2 n}\left(\frac{\varepsilon}{4}\right)\right)\right]=O\left(u_{n}^{2} \omega\left(u_{n}\right)\right), \\
& \int_{A_{2 n}} f^{1 / 2}\left(x, u_{n}\right) f^{1 / 2}(x, 0) d \nu<P_{u_{n}}^{1 / 2}\left(A_{2 n}\right) P^{1 / 2}\left(A_{2 n}\right)<C u_{n}^{2} \omega\left(u_{n}\right),
\end{aligned}
$$

поскольку, согласно (2.5), (3.14),

$$
\begin{aligned}
P_{u_{n}}\left(A_{2 n}\right) & =E\left[\left(\eta_{n}+1\right)^{2} \chi\left(A_{2 n}\right)\right]+P_{u_{n}}^{\mathrm{s}}(S) \\
& <2 E\left[\eta_{n}^{2} \chi\left(A_{2 n}\right)\right]+2 P\left(A_{2 n}\right)+P_{u_{n}}^{\mathbf{s}}(S)<C u_{n}^{2} \omega\left(u_{n}\right) .
\end{aligned}
$$

Применяя формулу Тейлора, получаем

$$
E\left[\xi_{n} \chi\left(B_{1 n}\right)\right]=2 J_{n 1}-J_{n 2}+J_{n 3},
$$

где $J_{n 1}=E\left[\eta_{n} \chi\left(B_{1 n}\right)\right], J_{n 2}=E\left[\eta_{n}^{2} \chi\left(B_{1 n}\right)\right], J_{n 3}=\frac{2}{3} E\left[\eta_{n}^{3}\left(1+\kappa \eta_{n}\right)^{-3} \chi\left(B_{1 n}\right)\right]$ и $0 \leqslant \kappa \leqslant 1$.

В силу (2.7), используя включение $B_{1 n} \subseteq B_{2 n}$, получаем

$$
J_{n 3}<C E\left[\left|\eta_{n}\right|^{3} \chi\left(B_{2 n}\right)\right]=O\left(u_{n}^{2} \omega\left(u_{n}\right)\right) .
$$

В силу (2.5), (3.14), (3.15) получаем

$$
\begin{aligned}
& J_{n 2}=\rho_{n}^{2}+O\left(u_{n}^{2} \omega\left(u_{n}\right)\right), \\
& J_{n 1}=-\frac{1}{2} J_{n 2}+O\left(u_{n}^{2} \omega\left(u_{n}\right)\right) .
\end{aligned}
$$

Из (3.16)-(3.19) следует (3.12).

Для любого $\delta>0$ имеем $|\ln x|<C_{\delta} x^{-\delta}$, если $x<1-\varepsilon, \varepsilon>0$. Отсюда, в силу условия $\mathrm{C}$, получаем

$$
\begin{aligned}
P_{u_{n}}\left(A_{1 n}\right) & \geqslant E\left[\xi_{n} \chi\left(A_{1 n}\right)\right] \geqslant-C_{\delta} \int_{S} f^{-\delta}\left(x, u_{n}\right) f^{1-\delta}(x, 0) \chi\left(A_{1 n}\right) d \nu \\
& =O\left(u_{n}^{2} \omega\left(u_{n}\right)\right) .
\end{aligned}
$$

Из (3.11), (3.25) и (3.31) следует (3.18). Доказательства (3.13)-(3.17) и (3.19), (3.20) аналогичны (3.18) и опускаются.

Докажем (3.21)-(3.23). Имеем $E[\tau]=0$ и, в силу (3.2), $E\left[|\tau| \chi\left(A_{3 n}\right)\right]<$ $C u_{n} E\left[\tau^{2} \chi\left(A_{3 n}\right)\right]<C u_{n} \omega\left(u_{n}\right)$, что означает (3.22).

В силу (2.2), (2.3), имеем

$$
\begin{aligned}
E\left(\eta_{n}-u_{n} \tau\right)^{2} & =u_{n}^{2} E \tau^{2}+E \eta_{n}^{2}-2 u_{n} E\left[\tau \eta_{n}\right] \\
& =\frac{1}{2} u_{n}^{2} I-2 u_{n} E\left[\tau \eta_{n}\right]+O\left(u_{n}^{2} \omega\left(u_{n}\right)\right)=O\left(u_{n}^{2} \omega\left(u_{n}\right)\right) .
\end{aligned}
$$


Из (3.3), (2.2) получаем

$$
\begin{aligned}
E\left[\tau \eta_{n}^{2} \chi\left(B_{3 n}\right)\right] & \leqslant 2 u_{n}^{2} E\left[|\tau|^{3} \chi\left(B_{3 n}\right)\right]+2 E\left[|\tau|\left(\eta_{n}-u_{n} \tau\right)^{2} \chi\left(B_{3 n}\right)\right] \\
& \leqslant C u_{n} \omega\left(u_{n}\right)+2 C u_{n}^{-1} E\left[\left(\eta_{n}-u_{n} \tau\right)^{2} \chi\left(B_{3 n}\right)\right] \\
& =O\left(u_{n} \omega\left(u_{n}\right)\right) .
\end{aligned}
$$

Из (3.21), (3.32), (3.33) следует, что

$$
\begin{aligned}
E_{u_{n}}\left[\tau \chi\left(B_{3 n}\right)\right] & =E\left[\tau\left(1+\eta_{n}\right)^{2} \chi\left(B_{3 n}\right)\right]+E_{u_{n}}\left[\tau \chi\left\{f\left(X_{1}, 0\right)=0\right\} \chi\left(B_{3 n}\right)\right] \\
& =2 E\left[\tau \eta_{n} \chi\left(B_{3 n}\right)\right]+O\left(u_{n} \omega\left(u_{n}\right)\right)+C u_{n}^{-1} P_{0, u_{n}}^{\mathrm{s}}(S) \\
& =\frac{1}{2} u_{n} I+O\left(u_{n} \omega\left(u_{n}\right)\right)
\end{aligned}
$$

что означает (3.19).

В силу (2.2), (3.3), получаем

$$
\begin{aligned}
E\left[\tau^{2}\left|\eta_{n}\right| \chi\left(B_{3 n}\right)\right] & \leqslant E\left[\tau^{2}\left|\eta_{n}-u_{n} \tau\right| \chi\left(B_{3 n}\right)\right]+u_{n} E\left[|\tau|^{3} \chi\left(B_{3 n}\right)\right] \\
& <\left(E\left[\tau^{4} \chi\left(B_{3 n}\right)\right]\right)^{1 / 2}\left(E\left(\eta_{n}-u_{n} \tau\right)^{2}\right)^{1 / 2}+O\left(\omega\left(u_{n}\right)\right) \\
& =O\left(\omega\left(u_{n}\right)\right) .
\end{aligned}
$$

В силу (2.2), (3.3), (3.35), получаем

$$
\begin{aligned}
E\left[\tau^{2} \eta_{n}^{2} \chi\left(B_{3 n}\right)\right]= & E\left[\tau^{2}\left(\eta_{n}-u_{n} \tau\right)^{2} \chi\left(B_{3 n}\right)\right]+2 u_{n} E\left[\left|\tau^{3} \eta_{n}\right| \chi\left(B_{3 n}\right)\right] \\
& +u_{n}^{2} E \tau^{4} \chi\left(B_{3 n}\right)<C u_{n}^{-2} E\left(\eta_{n}-u_{n} \tau\right)^{2} \\
& +C E\left[\tau^{2}\left|\eta_{n}\right| \chi\left(B_{3 n}\right)\right]+O\left(\omega\left(u_{n}\right)\right)=O\left(\omega\left(u_{n}\right)\right) .
\end{aligned}
$$

Из (3.35), (3.36) следует, что

$$
\begin{aligned}
& E_{u_{n}}\left[\tau^{2} \chi\left(B_{3 n}\right)\right]-E\left[\tau^{2} \chi\left(B_{3 n}\right)\right] \\
& \quad<E\left[\tau^{2}\left(\left(1+\eta_{n}\right)^{2}-1\right) \chi\left(B_{3 n}\right)\right]+C u_{n}^{-2} P_{u_{n}}^{s}(S)=O\left(\omega\left(u_{n}\right)\right) .
\end{aligned}
$$

Из (3.2), (3.37) следует (3.23). Доказательство леммы 3.4 закончено.

Доказательство теоремы 2.1 основано на следуюшей версии теоремы о вероятностях больших уклонений сумм независимых случайных величин. Пусть $Y_{1 n}, \ldots, Y_{n n}$ - независимые одинаково распределенные случайные величины, имеющие функцию распределения $G_{n}(x)$, и пусть $E Y_{1 n}=0, E Y_{1 n}^{2}=1$. Обозначим $S_{n}=Y_{1 n}+\cdots+Y_{n n}$ и положим $F_{n n}(x)=P\left\{S_{n}<x n^{1 / 2}\right\}$.

Сделаем следующее предположение.

Р. Сушествует последовательность $\varepsilon_{n}>0, \varepsilon_{n} \rightarrow 0, n \varepsilon_{n}^{2} \rightarrow \infty$ при $n \rightarrow \infty$, такая, что

$$
\begin{aligned}
& E \exp \left\{\varepsilon_{n} Y_{1 n}\right\}<C, \\
& E\left|Y_{1 n}\right|^{3}<C \varepsilon_{n}^{-1} \omega\left(\varepsilon_{n}\right) .
\end{aligned}
$$


Теорема 3.1. Пусть выполнено условие Р. Тогда для всех $x$ mакux, что $0<\delta \varepsilon_{n}<x n^{-1 / 2}<\varepsilon_{n}(1-\delta), \delta>0$, имеет место

$$
\ln \left(1-F_{n n}(x)\right)=-\frac{1}{2} x^{2}+O\left(n \varepsilon_{n}^{2} \omega\left(\varepsilon_{n}\right)\right),
$$

и если дополнительно $n \varepsilon_{n}^{2} \omega\left(\varepsilon_{n}\right) \rightarrow 0$ nри $n \rightarrow \infty$, mо

$$
\frac{1-F_{n n}(x)}{1-\Phi(x)}=1+O\left(\omega\left(\varepsilon_{n}\right)\right)
$$

при $n \rightarrow \infty$. Сходимость в (3.40) и (3.41) является равномерной на множестве всех последовательностей функиий распределения $G_{n}$, удовлетворяющих (3.38), (3.39) с данной константой $C$.

Доказательство теоремы 3.1 приведено в [10].

- Д о к аз а тель с т в о т е о ре мы 2.1. Имеем

$$
P\left\{N_{n s}-E N_{n \varepsilon s}>C_{n}, U_{n s}\right\}=P\left\{N_{n s}-E N_{n \varepsilon s}>C_{n} \mid U_{n s}\right\} P\left(U_{n s}\right)
$$

для $1 \leqslant s \leqslant 3$.

В силу (3.4), (3.25), имеем

$$
P\left(U_{n s}\right)=\left(1-P\left(A_{n s}\right)\right)^{n}=\overline{\exp }\left\{O\left(n u_{n}^{2} \omega\left(u_{n}\right)\right)\right\}=1+o(1) .
$$

Таким образом, доказательство (2.13) сводится к анализу вероятностей умеренных уклонений $P_{t}\left\{N_{n s}-E N_{n \varepsilon s}>C_{n} \mid U_{n s}\right\}$. Если имеет место событие $U_{n s}$, то случайные величины, входящие в сумму $N_{n s}-E N_{n \varepsilon s}$, ограничены и удовлетворяют (3.37). Из (2.7), (3.3) и

$$
E\left[\left|\xi_{n}\right|^{3} \chi\left(\left|\xi_{n}\right|<\varepsilon\right)\right]<32 E\left[\left|\eta_{n}\right|^{3} \chi\left(\left|\eta_{n}\right|<2 \varepsilon\right)\right]
$$

для $0<\varepsilon<\frac{1}{3}$ следует, что (3.28) имеет место, когда $Y_{i n}$ равно $\xi_{n i} \chi\left(B_{n 1 i}\right), \eta_{n i} \chi\left(B_{n 2 i}\right)$ и $\tau_{i} \chi\left(B_{n 3 i}\right)$. Таким образом, в силу теоремы 3.1 , мы получаем (2.13). Равенство (2.14) следует из замечания 3.1 и аналогичных оценок.

Остается доказать (2.11). Обозначим $Y_{n i}=\bar{\xi}_{n i}=\left(2 \rho_{n}\right)^{-1}\left(\xi_{n i}+2 \rho_{n}^{2}\right)$, $1 \leqslant i \leqslant n$. Тогда (3.39) следует из (3.44) и оценок, аналогичных (3.31). Неравенство (3.38) следует из (2.9). Теорема 2.1 доказана.

\section{СПИСОК ЛИТЕРАТУРЫ}

1. Алешкявичене $A . K$. Вероятности больших уклонений для $U$-статистик и функционалов фон Мизеса. - Теория вероятн. и ее примен., 1990, т. 35, в. 1, с. 1-13.

2. Архангельский A.H. Нижние границы для вероятностей больших уклонений сумм независимых случайных величин. - Теория вероятн. и ее примен., 1989, т. 34, в. 4 , c. $625-635$.

3. Bahadur R. R. On the asymptotic efficiency of tests and estimates. - Sankhyā, 1960, v. 22 , p. $229-252$. 
4. Боровков A.A., Могульский $A . A$. Большие уклонения и проверка статистических гипотез. - Труды Ин-та математики СО РАН, т. 19. Новосибирск: Наука, $1992,222 \mathrm{c}$.

5. Боровков $A . A$., Могульский $A$. $A$. Большие уклонения и статистический принцип инвариантности. - Теория вероятн. и ее примен., 1992, т. 37, в. 1, с. 11-18.

6. Chernoff $H$. A measure of asymptotic efficiency for tests of a hypothesis based on sums of observations. - Ann. Math. Statist., 1952, v. 23, p. 493-507.

7. Ermakov M.S. Asymptotic minimaxity of usual goodness of fit tests. - Probability Theory and Mathematical Statistics. Proceedings of the 5th Vilnius Conference. V. I. Ed. by B. Grigelionis et al. Zeist/Vilnius: VSP/Mokslas, 1991, p. 323-331.

8. Ермаков M. С. Большие уклонения эмпирических мер и проверка гипотез. - Зап. научн. семин. ЛОМИ, 1993, т. 207, с. 37-60.

9. Ермаков М. С. Асимптотическая минимаксность критериев типа Колмогорова и омега-квадрат. - Теория вероятн. и ее примен., 1995, т. 40, в. 1, с. 54-67.

10. Ермаков М.С. О нижней границе для точной асимптотики вероятностей больших уклонений статистических оценок. - Проблемы передачи информации, 1999, т. 35 , № 3, c. $46-60$.

11. Гринвуд П. Е., Ибрагимов И. А. Об асимптотически эффективном оценивании по Бахадуру. - Докл. РАН, 1993, т. 32, № 1, с. 5-7.

12. Hajek $J$. Local asymptotic minimax and admissibility in estimation. - Proceedings of the Sixth Berkeley Symposium on Mathematical Statistics and Probability. V. I: Theory of Statistics. Berkeley: Univ. California Press, 1972, p. 175-194.

13. Hodges J. L., Lehmann E. L. The efficiency of some nonparametric competitors of the $t$-test. - Ann. Math. Statist., 1956, v. 27, p. 324-335.

14. Inglot T., Kallenberg W. C. M., Ledwina T. Strong moderate deviation theorems. Ann. Probab., 1992, v. 20, № 2, p. 987-1003.

15. Jurecková J., Kallenberg W. C. M., Veraverbeke W. Moderate and Cramér-type deviation theorems for $M$-estimators. - Statist. Probab. Lett., 1988, v. 6, p. 191-199.

16. Kallenberg W. C. M. Intermediate efficiency, theory and examples. - Ann. Statist., 1983, v. 11, № 1, p. 170-182.

17. Kourouklis $S$. On the relation between Hodges-Lehman efficiency and Pitman efficiency. - Canad. J. Statist., 1989, v. 17, № 3, p. 311-318.

18. Kourouklis $S$. A relation between the Chernoff index and the Pitman efficiency. Statist. Probab. Lett., 1990, v. 9, № 5, p. 391-395.

19. Le Cam L. Asymptotic Methods in Statistical Decision Theory. New York: SpringerVerlag, 1986, $742 \mathrm{p}$.

20. Oosterhoff J., van Zwet W.R. A note on contiguity and Hellinger distance. - Contributions to Statistics. Hajek Memorial Volume. Ed. by J. Jurecková. Dordrecht: Reidel, 1979, p. 157-166.

21. Radavicius $M$. From asymptotic efficiency in minimax sense to Bahadur efficiency. New Trends in Probability and Statistics. V. 1. Ed. by V. Sazonov and T. Shervashidze. Zeist/Vilnius: VSP/Mokslas, 1991, p. 629-635.

22. Strasser H. Mathematical Theory of Statistics. Berlin-New York: de Gruyter, 1985, $492 \mathrm{p}$.

23. Wieand H.S. A condition under which the Pitman and Bahadur approaches to efficiency coincide. - Ann. Statist., 1976, v. 4, p. 1003-1011.

24. Wolfowitz J. Asymptotic efficiency of the maximum likelihood estimator. - Теория вероятн. и ее примен., 1965, т. 10, в. 2, с. 267-281. 